\title{
Phenomenology of first-order dark-state phase transitions
}

\author{
Dietrich Roscher, ${ }^{1,2}$ Sebastian Diehl, ${ }^{1}$ and Michael Buchhold ${ }^{3}$ \\ ${ }^{1}$ Institut für Theoretische Physik, Universität zu Köln, D-50937 Cologne, Germany \\ ${ }^{2}$ Department of Physics, Simon Fraser University, Burnaby, British Columbia, Canada V5A 1S6 \\ ${ }^{3}$ Department of Physics and Institute for Quantum Information and Matter, California Institute of Technology, \\ Pasadena, California 91125, USA
}

(Received 10 April 2018; revised manuscript received 2 July 2018; published 26 December 2018)

\begin{abstract}
Dark states are stationary states of a dissipative, Lindblad-type time evolution with zero von Neumann entropy, therefore representing examples of pure steady states. Nonequilibrium dynamics featuring a dark state recently gained a lot of attraction since their implementation in the context of driven-open quantum systems represents a viable possibility to engineer unique, pure states. Inspired by recent experimental progress with ultracold Rydberg ensembles, we analyze a driven many-body spin system, which displays a mean-field bistability between a dark steady state and a mixed steady state. As a function of the driving strength one observes a discontinuous phase transition that connects the zero entropy (dark) state with a finite entropy (mixed) state. The transition is characterized by a jump of the von Neumann entropy from zero to a finite value, which is of genuine nonequilibrium character. We analyze the relevant long wavelength fluctuations driving this transition by means of the renormalization group. This allows us to approach the nonequilibrium dark-state transition and identify similarities and clear differences to common, equilibrium phase transitions, to establish the phenomenology for a first-order dark-state phase transition, and to relate it to the dynamics in driven dissipative Rydberg ensembles.
\end{abstract}

DOI: 10.1103/PhysRevA.98.062117

\section{INTRODUCTION}

\section{A. General Motivation}

Understanding the dynamics at first-order phase transitions, which is characterized by large fluctuations between two or more macroscopic and metastable order parameter configurations, represents a general challenge for theoretical physics $[1,2]$. The interest in such dynamics has only recently started growing again, triggered by the observation that a diverse set of driven dissipative many-body systems feature optical bistability in regimes of strong drive and dissipation, ranging from driven Rydberg ensembles [3-5] to nonlinear photon lattices [6-8], semiconductor microcavities [9], and cavity QED with cold atoms [10-15].

On the microscopic scale, the steady states of these driven, bistable systems are rather determined by a flux equilibrium between external drive and dissipative forces than from detailed balance between individual states [16-18]. Typical sources of dissipation, e.g., cavity photon loss, spontaneous emission, and dephasing, share, however, that they imprint a finite, constant noise level on the atom or photon degrees of freedom. Going from the microscopic to the macroscopic scale, this constant noise level typically leads to the emergence of detailed balance between different, macroscopic many-body states $[16,19,20]$. In particular for optical bistability, it establishes detailed balance between the different metastable states. The corresponding thermodynamic phase transition happens at a fixed, finite von Neumann entropy and turns the bistability into an effective, dissipative equilibrium first-order phase transition [8,21-24], well captured by the equilibrium Ising model.

Here we explore a realization of optical bistability in driven Rydberg ensembles that prohibits the emergence of detailed balance on any scale and features genuinely nonequilibrium dynamics from microscopic to macroscopic scales. The key for maintaining nonequilibrium conditions up to thermodynamic scales is the presence of a unique, robust dark state, i.e., a pure steady state without any statistical fluctuations [25-28], in the bistable regime. By explicitly varying the drive strength versus the dissipation, the Rydberg system establishes a bistability regime, in which the asymptotic stationary state is either a mixed state with a nonvanishing von Neumann entropy $S>0$, or a dark state with zero entropy $S=0$. The vanishing entropy of the dark state completely suppresses any fluctuations and prohibits the establishment of detailed balance in the bistable regime at any scale, rendering the dynamics inherently nonequilibrium. Including the effect of long-wavelength fluctuations, the region of bistability in parameter space shrinks and is confined towards a single coexistence point in the thermodynamic limit, at which a first-order dark-state phase transition is expected [5,29].

The absence of fluctuations in one phase and the presence of large fluctuations in the other brings phase transitions between a dark state and a mixed state into contact with nonequilibrium statistical physics, where classical steady states with zero entropy are known as absorbing states [5,29-33]. Continuous phase transitions into absorbing states have been extensively studied theoretically since they are believed to model a diverse set of dynamical processes such as disease spreading, the evolution of forest fires, percolation processes, and population dynamics [34-37]. A key feature to these models is a phase characterized by a fluctuationless, zero-entropy state, separated from a fluctuating phase of nonzero entropy. This prohibits the presence of a temperature scale and makes the phase transition into absorbing states a genuine nonequilibrium transition $[38,39]$. 
The dynamics in the bistability region between a dark state and a mixed steady state and at the first-order darkstate phase transition is very special in several respects. Phenomenologically, the transition from one thermodynamic phase to another via a first-order transition is understood in terms of the nucleation of small droplets and their subsequent growth to a macroscopic size $[1,2,40]$. In the dark-state framework this implies that large regions of zero entropy as well as nonvanishing entropy coexist close to a first-order transition, a genuinely nonequilibrium circumstance. Exactly at the transition, however, both the zero and the finite entropy phase coexist, which translates to the coexistence of pure as well as mixed steady states in a density matrix picture. Even the classical counterparts, first-order phase transitions into absorbing states, are hardly understood on a qualitative level and believed to be very rare in higher dimensions and even impossible in one dimension [41-44]. Due to the absence of universality in these setups, even slight changes in the microscopic model can have drastic consequences for the resulting phase structure (cf., e.g., $[44,45]$ ), further obstructing a general understanding of these phenomena.

Experimental realizations of absorbing state phase transitions using "quantum ingredients" have been proposed in the framework of driven Rydberg systems very recently for both incoherent and coherent drive $[29,33,46]$. These absorbing states have, however, not been explicitly identified as dark states as a large part of the work was carried out in the classical limit of strong dephasing [5]. We build up on these proposals and study the dynamics of coherently driven Rydberg ensembles, for which the notion of a dark-state phase transition is most appealing.

The intention of this work is to establish the phenomenology for a first-order dark-state transition in dependence of spatial dimensionality. We also introduce a functional renormalization group [47-49] based approach, tailored to investigate long-wavelength dynamics of large order parameter fluctuations in a nonequilibrium setting to the currently developing theoretical toolbox for driven dissipative dynamics [10,5052]. This approach works in both low and high dimensions and allows us to resolve the rather complex but also very rich and fascinating dynamics close to a first-order phase transition, as well as a fluctuation induced second-order phase transition, which we highlight on the following pages.

The dark state can be understood as a simple ferromagnetic product state and is thus particularly well suited for the investigation of the phase transition. As we will show, this allows us to construct an order parameter field that is free of any fluctuations, classical and quantum, when the system is in the dark state. Thus the defining property of a dark state, i.e., the absence of statistical fluctuations, is not masked by the remaining quantum fluctuations of the order parameter. The quantum nature of the system nevertheless comes crucially into play at two different stages. First, nonvanishing coherences in the local spin density matrix are necessary to make a discontinuous transition in this system at all possible [5] and second, the absence of any statistical fluctuation scale requires a pure state.

In order to analyze the steady state properties and the dynamics in the bistable regime, we identify the local density of atoms in the Rydberg state as the order parameter. Its expectation value and fluctuations vanish exactly for a dark state. Its dynamics will be described in terms of an effective, nonequilibrium field theory, which we solve via a semianalytical approach based on the functional renormalization group (fRG). Relating the obtained results with the phenomenology of thermal first-order transitions allows us to extract a number of physical insights regarding the nonequilibrium setting. We benchmark these findings with numerically exact results in one spatial dimension, thereby establishing the method as a general tool to study first-order nonequilibrium phase transitions.

\section{B. Summary of results}

Including the effect of spatial fluctuations in the absence of detailed balance, we demonstrate that both a dark-state phase as well as a mixed state phase can be stabilized in the presence of nonequilibrium noise. We quantitatively determine the location of the phase boundary and the nature of the phase transition as a function of dimensionality and the strength of the noise level. We observe two distinct scenarios, a sharp first-order phase transition, accompanied with a discontinuous jump of the order parameter on the one hand and a fluctuation induced, second-order phase transition, for which spatial fluctuations have softened the evolution of the order parameter towards a continuous phase transition on the other hand. Both phase transitions are clearly distinguishable from each other and from possible equilibrium counterparts by the unique behavior of the order parameter as well as its fluctuations.

Starting from the quantum master equation for the driven dissipative Rydberg setup [22,23,29,33,53,54], we introduce the density of atoms in the Rydberg state $v_{X} \geqslant 0, X=(x, t)$ as the order parameter field for the dark-state transition in $d$ spatial dimensions. In the bistable regime, the order parameter undergoes diffusive relaxation in a double-well potential $V$. Its dynamics follows the fundamental Langevin equation

$$
\begin{gathered}
\partial_{t} v_{X}=D \nabla^{2} v_{X}-V^{\prime}\left(v_{X}\right)+\xi_{X}, \\
V\left(v_{X}\right)=\frac{\Delta}{2} v_{X}^{2}+\frac{\mu}{3} v_{X}^{3}+\frac{\lambda}{4} v_{X}^{4},
\end{gathered}
$$

with a multiplicative, Gaussian noise $\xi_{X}$ of zero mean $\left\langle\xi_{X}\right\rangle=$ 0 and variance $\left\langle\xi_{X} \xi_{Y}\right\rangle=\delta(X-Y) \gamma v_{X}$. The double-well potential $V$ is illustrated in Fig. 1. It has two minima at $v_{X}=0$ and $v_{X}=v_{f}>0$, which are separated by a barrier that is maximal at $v_{X}=v_{\max }$ and can only be overcome by the field due to noise induced activation or spatial fluctuations.

The absence of both common symmetries and a welldefined scaling dimension for $v_{X}$ in a double-well potential with multiplicative noise separates the Langevin equation (1.1) from other nonequilibrium settings [19,34-37,55] and requires a nonperturbative approach. Considering the general importance of fluctuations on all length scales at discontinuous phase transitions, we establish a minimal, yet sufficient fRG approach suitable for nonequilibrium first-order phase transitions. It evolves both the potential and the noise kernel in a nontrivial way, which turns out to be of major importance for the resolution of droplet formation and density fluctuations at the first-order phase transition, and allows us to develop a clear picture of the dynamics. 


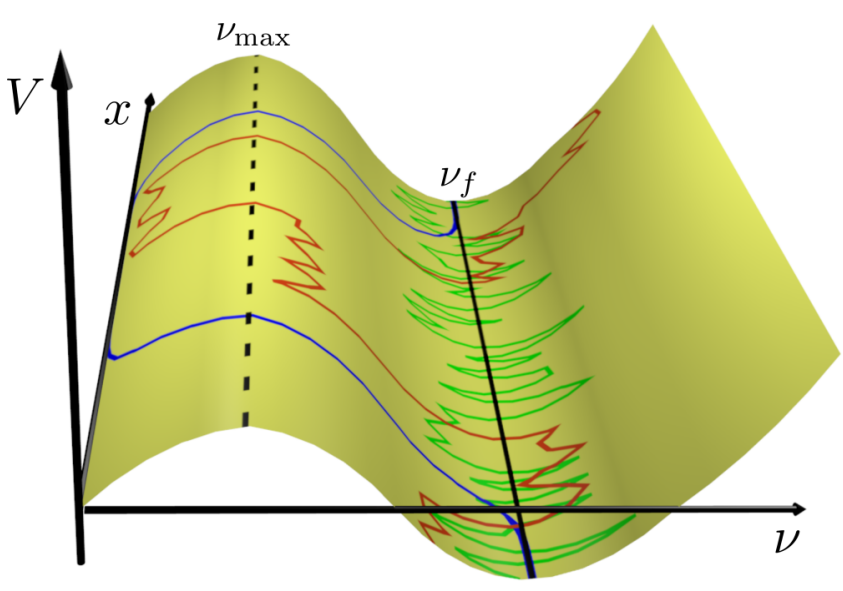

FIG. 1. Portrait of prototypical inhomogeneous configurations of the coarse-grained excitation density $v_{x}$ in one spatial dimension (labeled with $x$ ) that fluctuate about the nontrivial minimum at $v_{x}=$ $v_{f}$. At each point in space $x$ the field has a value $v_{x}$ and experiences the potential $V\left(v_{x}\right)$, which is maximal at $V\left(v_{\max }\right)$. Perturbatively small amplitude oscillations (green) do not probe global properties of $V$. In contrast, dropletlike saddle-point solutions (blue) or strongly fluctuating configurations (red) are crucial to understand the dominant behavior at the first-order and the second-order phase transition, as discussed below.

In higher dimensions $d>1$, the potential barrier persists under the fRG evolution up to the largest scales and vanishes only in the limit of long wavelengths, establishing a single minimum that jumps when passing the transition. This is a clear indication of a first-order transition induced by the nucleation and growth of metastable field configurations, socalled droplets. The droplets interpolate smoothly between the two phases (blue configuration in Fig. 1) and thus require the presence of a potential barrier on all scales [2,40].

The droplet regime is heralded at a very sharp length scale by the observation of both the onset of the mentioned potential evolution as well as a sudden deformation of the noise kernel. The deformed noise establishes a bimodal structure, indicating strongly pronounced order parameter fluctuations between the two phases, see Fig. 10. Both observations are in accordance with a first-order phase transition driven by the formation of droplets of well-defined extent [1,2,40], which defines a clearly observable scale. At this scale droplets nucleate and perform sudden jumps from one phase to the other, leading to an increased fluctuation rate of the metastable field configurations. It is observable by strongly increased density fluctuations and inhomogeneous real space patterns. In contrast, in $d=1$, neither a sharp length scale for the onset of the evolution nor a pronounced structure of the noise kernel can be identified, pointing out the crucial difference between fluctuations leading to first- or second-order phase transitions.

At the first-order transition, we observe that both the potential and the noise kernel $\chi=V=0$ become completely flat for an extended region of field configurations $0 \leqslant v_{X} \leqslant v_{f}$. This is identified with the emergence of an extensive number of stationary, noiseless field configurations, which is a unique mechanism to ensure the coexistence of a zero entropy (darkstate) phase and a finite entropy phase at the same point. In a master equation framework this remarkable observation hints towards an extensive number of dark states at the coexistence point, each corresponding to a specific droplet configuration. This is contrasted by the common observation of two degenerate steady states at a dissipative equilibrium coexistence point $[7,56]$.

In one dimension, the dark-state transition becomes second order. Here spatial fluctuations are strongly enhanced and can overcome the potential barrier, which leads to a continuous evolution of $v_{X}$ at the phase transition. This can be directly traced with the fRG flow of the potential. Fluctuations on intermediate wavelengths render the initial double well convex and establish a single, global minimum for $V$. The dark and mixed state then are no longer separated by a potential barrier and the long-wavelength modes fluctuate randomly between the two solutions (red configuration in Fig. 1). Scaling dynamics, however, persists up to the largest length scales and the single potential minimum evolves towards zero under the influence of long distance modes, thus undergoing a continuous transition [57]. The absence of common symmetries and a well-defined scaling dimension promotes this second-order transition to a candidate for a so far unexplored nonequilibrium universality class.

\section{DARK-STATE SPIN MODEL}

The opportunity to precisely manipulate entire ensembles of atomic spins with quantum optical tools, such as coherent drive and pump lasers [16,58-62], as well as with dissipative jump operators via reservoir engineering [63-66], pushed forward the search for robust, many-body dark states. The latter are pure states $\rho_{\mathrm{DS}}=|\mathrm{DS}\rangle\langle\mathrm{DS}|$, which are exact, stationary zero modes $\mathcal{L} \rho_{\mathrm{DS}}=0$ of the quantum master equation $\partial_{t} \rho=$ $\mathcal{L} \rho$. Once a dark state is reached during the time evolution, the system will remain forever in this particular state and the dynamics has terminated. In this sense, $\rho_{\mathrm{DS}}$ represents the quantum mechanical analog of a classical absorbing state, for which both the deterministic time evolution as well as reservoir induced fluctuations vanish. The von Neumann entropy $S(\rho)=-\operatorname{tr}(\rho \ln \rho)$ of a dark state is always zero $S\left(\rho_{\mathrm{DS}}\right)=$ $-\ln 1=0$.

Similar to a fluctuationless absorbing state, dark states are very clean representatives of steady states, which feature the complete absence of statistical (but not necessarily quantum) fluctuations. The mere existence of a dark-state $\rho_{\mathrm{DS}}$ in a given model does, however, not imply that $\rho_{\mathrm{DS}}$ is the only attractor of the dynamics $[5,29]$. Under certain conditions, which we will elaborate on in more detail below, it may be that in addition to a unique dark state, there exists another, mixed steady state $\rho_{\mathrm{SS}}=\rho_{\mathrm{MS}}=\sum_{\alpha} p_{\alpha}\left|\psi_{\alpha}\right\rangle\left\langle\psi_{\alpha}\right| \neq \rho_{\mathrm{DS}}$, such that each initial state $\rho(t=0) \neq \rho_{\text {DS }}$ evolves towards $\rho_{\text {SS }}$ asymptotically, $\rho(t) \stackrel{t \rightarrow \infty}{\rightarrow} \rho_{\text {SS }}$. This mixed state has per definition nonvanishing von Neumann entropy $S\left(\rho_{\mathrm{MS}}\right)=-\sum_{\alpha} p_{\alpha} \ln p_{\alpha}>0$.

Below we introduce the quantum master equation for a minimalistic model of a dark-state phase transition, which can be realized with cold Rydberg atoms. We show that this model is equivalent to a coarse-grained field theory for a real density field, which diffuses through a double-well potential under the influence of a multiplicative noise. This is one of the most simple field theories one can imagine for a nonequilibrium first-order phase transition, fulfilling the criterion of bistability 
supplemented with the presence of spatial fluctuations and a nonthermal noise.

\section{A. Quantum master equation}

One key mechanism to achieve true nonequilibrium dynamics in driven Rydberg ensembles is the exploitation of the so-called antiblockade mechanism [46,67-71]. Excitations of ground state atoms into the Rydberg state are thereby facilitated in the vicinity of other Rydberg-excited atoms via the resonant interplay of interactions and drive. We consider a minimal model for this dynamics, which features the transition from a dark state towards a fluctuating steady state. It has been proposed in Refs. [5,29] and is inspired by recent experiments with driven Rydberg ensembles [60,70,72-74]. After discussing its basic properties, we map this model to the more convenient and fundamental stochastic Langevin equation (1.1), which will then be analyzed in a field theoretic framework. The relation of this Langevin equation to other theoretical approaches to driven Rydberg systems and to experiments $[71,73]$ is discussed afterwards.

We consider the time evolution of an ensemble of spins on a $d$-dimensional square lattice subject to coherent drive. Each site, labeled with $l$, hosts a single spin- $\frac{1}{2}$, which represents the ground $|g\rangle_{l} \equiv|\downarrow\rangle_{l}$ or the excited $|e\rangle_{l} \equiv|\uparrow\rangle_{l}$ state of a Rydberg atom. The local operator Hilbert space is spanned by the Pauli-operators $\sigma_{l}^{x, y, z}$ and the unit operator $\mathbb{1}_{l}$. The spins are subject to a coherent drive laser with Rabi frequency $\Omega$ and detuning $\delta=-V_{\mathrm{nn}}$, where $V_{\mathrm{nn}}$ is the nearest neighbor Rydberg repulsion. Setting the Rydberg repulsion to be by far the strongest scale, transitions between the ground and the Rydberg state are only resonant in the vicinity of another excited atom, while they are far off-resonance in the absence of nearby Rydberg excitations. In order to understand the nature of the "clean" phase transition in the absence of any offresonant processes, we project the dynamics onto the sector of resonant transitions. This yields the Hamiltonian [5,29]

$$
H=\Omega \sum_{l} \Pi_{l} \sigma_{l}^{x}
$$

Here $\Pi_{l}$ is the projector onto the states with at least one upspin in the neighborhood of site $l$,

$$
\Pi_{l}=\mathbb{1}-\prod_{m \operatorname{nn} l}\left(\mathbb{1}-n_{m}\right), \quad \text { with } n_{m}=\frac{\sigma_{m}^{z}+1}{2} .
$$

For a state $|\psi\rangle$ one finds $\Pi_{l}|\psi\rangle=0$ if $n_{m}|\psi\rangle=0$ for all nearest neighbors (nn) $m$ of $l$ and $\Pi_{l}|\psi\rangle=|\psi\rangle$ else.

Highly excited Rydberg states spontaneously emit a photon and decay incoherently into the ground state with a rate $\gamma$. The combination of Hamiltonian dynamics and incoherent decay is described by the master equation

$$
\partial_{t} \rho=\mathcal{L} \rho=i[\rho, H]+\gamma \sum_{l}\left(\sigma_{l}^{-} \rho \sigma_{l}^{+}-\frac{1}{2}\left\{n_{l}, \rho\right\}\right)
$$

with the common spin ladder operators $\sigma_{l}^{ \pm}$and the Liouvillian superoperator $\mathcal{L}$ summarizing the right-hand side.
The quantum master equation features one dark state independently of the choice of the coupling constants $\{\Omega, \gamma\}$. This is the ferromagnetic spin-down state

$$
\rho_{\text {DS }}=\left|\psi_{\downarrow}\right\rangle\left\langle\psi_{\downarrow}\right|, \quad \text { with }\left|\psi_{\downarrow}\right\rangle=\prod_{l}|\downarrow\rangle_{l},
$$

and it is easy to see that $\mathcal{L} \rho_{\mathrm{DS}}=0$. While this state, however, is present throughout the entire parameter regime, we will show that there exists a large domain $\mathcal{A}$ in parameter space, for which $\rho_{\mathrm{DS}}$ is not attracting the dynamics and becomes fully repulsive in the thermodynamic limit. In other words, for $(\Omega, \gamma) \in \mathcal{A}$ any initial state $\rho_{0}$ that is different from $\rho_{\text {DS }}$ will evolve towards a steady state $\rho_{\text {SS }} \neq \rho_{\text {DS }}$. Within this domain, the dark state represents an isolated state, which can neither be reached nor left dynamically. Crossing the boundary of $\mathcal{A}$ is connected to a phase transition from $\rho_{\mathrm{SS}} \neq \rho_{\mathrm{DS}}$ towards $\rho_{\mathrm{SS}}=\rho_{\mathrm{DS}}$.

\section{B. Rydberg density Heisenberg-Langevin equation}

The dark-state $\rho_{\text {DS }}$ is an eigenstate of the local density

$$
n_{l} \rho_{\mathrm{DS}}=0,
$$

which corresponds to a vanishing density of Rydberg excitations. Since for integer $m$ one finds $n_{l}^{m}=n_{l}$, this implies that all moments of the density are zero in the dark state. It is a fluctuationless state with respect to $n_{l}$. For any mixed state, $\left\langle n_{l}\right\rangle>0$ for some $l$ and thus $\left\langle n_{l}\right\rangle$ serves well as an order parameter for the dark-state transition. In order to investigate its dynamics, we derive now the corresponding field theory. We take a different route than Refs. [5,29] and adiabatically eliminate $\sigma_{l}^{x, y}$, which appear to be gapped throughout the whole parameter regime on the operator level. This results in an effective, coarse-grained Heisenberg-Langevin equation for $n_{l}$. The final result is not different but the derivation more transparent than previous approaches.

The Heisenberg-Langevin equations for the spin operators $o_{\alpha}=\sigma_{l}^{x}, \sigma_{l}^{y}, n_{l}$ are operator valued stochastic differential equations $\partial_{t} o_{\alpha}=\mathcal{D}_{\alpha}+\xi_{\alpha}$. They contain a drift term $\mathcal{D}_{\alpha}$ and a quantum noise $\xi_{\alpha}$ [75]. The drift $\mathcal{D}_{\alpha}$ describes the action of the adjoint superoperator on $o_{\alpha}$,

$$
\mathcal{D}_{\alpha}=\mathcal{L}^{*} o_{\alpha}=i\left[H, o_{\alpha}\right]+\gamma \sum_{l}\left(\sigma_{l}^{+} o_{\alpha} \sigma_{l}^{-}-\frac{1}{2}\left\{n_{l}, o_{\alpha}\right\}\right)
$$

The quantum noise $\xi_{\alpha}$ describes the evolution of fluctuations of $o_{\alpha}$. It has zero mean $\left\langle\xi_{\alpha}\right\rangle_{\text {noise }}=0$, and an operator valued noise kernel $\chi_{\alpha \beta}=\left\langle\xi_{\alpha} \xi_{\beta}\right\rangle_{\text {noise }}$, where the noise average has to be understood as the average over all bath degrees of freedom (cf. [75]). It is determined via the Einstein relation [76]

$$
\begin{aligned}
\chi_{\alpha \beta} & =\left\langle\partial_{t}\left(o_{\alpha} o_{\beta}\right)-\left(\left(\partial_{t} o_{\alpha}\right) o_{\beta}+o_{\alpha} \partial_{t} o_{\beta}\right)\right\rangle_{\text {noise }} \\
& =\left\langle\mathcal{L}^{*}\left(o_{\alpha} o_{\beta}\right)-\left(\mathcal{D}_{\alpha} o_{\beta}+o_{\alpha} \mathcal{D}_{\beta}\right)\right\rangle_{\text {noise }} .
\end{aligned}
$$

The operator expectation value $\left\langle o_{\alpha}\right\rangle$ is then defined as the combination of noise average and system average, i.e.,

$$
\left\langle o_{\alpha}\right\rangle=\operatorname{Tr}\left(\rho\left\langle o_{\alpha}\right\rangle_{\text {noise }}\right) .
$$


The Heisenberg-Langevin equations for the local spin operators read as

$$
\begin{gathered}
\partial_{t} n_{l}=-\gamma n_{l}+\Omega \sigma_{l}^{y} \Pi_{l}+\xi_{l}^{n} \\
\partial_{t} \sigma_{l}^{x}=-\Omega\left(\sigma_{l}^{y} s_{l}+s_{l} \sigma_{l}^{y}\right)-\frac{\gamma}{2} \sigma_{l}^{x}+\xi_{l}^{x}, \\
\partial_{t} \sigma_{l}^{y}=\Omega\left(\sigma_{l}^{x} s_{l}+s_{l} \sigma_{l}^{x}\right)-\frac{\gamma}{2} \sigma_{l}^{y}+\xi_{l}^{y}-2 \Omega\left(2 n_{l}-1\right) \Pi_{l} .
\end{gathered}
$$

We introduced the operator $s_{l}=\sum_{m \mathrm{nn} l}\left(\Pi_{m}-1\right) \sigma_{m}^{x}$. The Hermitian noise operators $\xi_{l}^{n, x, y}$ have a flat power spectrum, i.e., they are $\delta$ correlated in time, due to the Markovian nature of the master equation.

Defining the noise vector $\xi_{l}=\left(\xi_{l}^{n}, \xi_{l}^{x}, \xi_{l}^{y}\right)$ on each lattice site, the Einstein relation (2.7) yields the noise kernel

$$
\chi_{l, m}=\left\langle\boldsymbol{\xi}_{l}^{\dagger} \boldsymbol{\xi}_{m}\right\rangle_{\text {noise }}=\gamma \delta_{l, m}\left(\begin{array}{ccc}
n_{l} & \sigma_{l}^{+} & -i \sigma_{l}^{+} \\
\sigma_{l}^{-} & 1 & -i \\
i \sigma_{l}^{-} & i & 1
\end{array}\right) .
$$

The presence of a dark state is reflected in the HeisebergLangevin equations. For average values $\left\langle n_{l}\right\rangle=0$ for all $l$, the projectors $\left\langle\Pi_{l}\right\rangle=0$ vanish equally well and thus the lattice sites decouple. This implies $\left\langle\sigma_{l}^{ \pm, x, y}\right\rangle=0$ for all $l$, such that the deterministic parts of Eqs. (2.9)-(2.11) vanish and the noise kernel $\chi_{l, m}$ is only nonzero in the $x-y$ sector [77]. This demonstrates that the property $\left\langle n_{l}\right\rangle=0, \forall l$,

(1) is always a possible solution for the HeisenbergLangevin equations (2.9)-(2.12),

(2) is the necessary and sufficient condition for reaching the dark-state $\rho_{\mathrm{DS}}=\left|\psi_{\downarrow}\right\rangle\left\langle\psi_{\downarrow}\right|$,

(3) leads to the absence of any density fluctuations in Heisenberg-Langevin equations.

The densities $n_{l}$ thus represent reliable order parameters for the transition from a fluctuating, mixed steady state $\left(n_{l} \neq 0\right)$ towards a fluctuationless dark state $\left(n_{l}=0\right)$. The fact that such a transition cannot be described in terms of an effective equilibrium statistical mechanics approach is reflected in the Heisenberg-Langevin framework by the multiplicative density noise $\xi_{l}^{n} \sim \sqrt{n_{l}}$. In the vicinity of $n_{l}=0$ this cannot be mapped to an effective, temperaturelike noise kernel.

In order to derive an effective Heisenberg-Langevin equation for the density alone, we follow the common procedure and adiabatically eliminate the fast variables $\sigma_{l}^{x, y}$ from the set of equations (2.9)-(2.11). After some algebra and the restriction to nearest neighbor couplings, one finds the operator equation for the spin-up density

$$
\partial_{t} n_{l}=-\gamma n_{l}-2 \Omega^{2} \Pi_{l}\left(\mathcal{K}^{-1}\right)_{l, j}\left(2 n_{j}-1\right) \Pi_{j}+\tilde{\xi}_{l}^{n},
$$

with the (regular) retarded operator

$$
\mathcal{K}_{l, j}=\delta_{l, j} \frac{\gamma}{2}+\delta_{j, \mathrm{nn} l} \frac{32 \Omega^{2}}{\gamma^{2}}\left(1-\Pi_{j}\right)+i 0^{+}
$$

and an effective noise $\tilde{\xi}_{l}^{n}$ with kernel

$$
\left\langle\xi_{l}^{n} \xi_{m}^{n}\right\rangle_{\text {noise }}=\delta_{l, m} \gamma n_{l}+\gamma \Omega^{2} \Pi_{l}\left(\mathcal{K}^{-2}\right)_{l, m} \Pi_{m} .
$$

These equations are valid on timescales larger than the relaxation time $t>\gamma^{-1}$ of the operators $\sigma_{l}^{x, y}$.
In order to analyze the dynamics described by the Heisenberg-Langevin equation for the operators $n_{l}$ (2.13) with the noise kernel (2.15), we perform a long-wavelength analysis of the corresponding Langevin equation for the coarse-grained density expectation value $v(x, t)$, where $x$ is a continuous variable in $d$-dimensional space and $t$ is time. We define $v(x, t)$ as the dimensionless excitation density per site in a $d$-dimensional, small but macroscopic volume, which contains $N_{x} \gg 1$ lattice sites centered around the coordinate $x$, i.e.,

$$
v(x, t)=\frac{1}{N_{x}} \sum_{j=1}^{N_{x}}\left\langle n_{j}\right\rangle(t) .
$$

The Langevin equation for $v_{X}=v(x, t)$ is obtained by evaluating the operator expectation on the right-hand side of Eq. (2.13) within three crucial coarse-graining steps:

(1) A site decoupling mean-field approach, i.e., $\left\langle n_{j} n_{l}\right\rangle(t)=\left\langle n_{j}\right\rangle(t)\left\langle n_{l}\right\rangle(t)$ for different sites $l \neq j$.

(2) The temporal decoupling on identical lattice sites, i.e., $\left\langle n_{l} n_{l}\right\rangle=\left\langle n_{l}\right\rangle(t)\left\langle n_{l}\right\rangle\left(t^{\prime}\right)$ if $t$ and $t^{\prime}$ are not identical. This is important in order to evaluate the product $\Pi \tilde{\mathcal{K}}^{-1} \Pi$, for which $\tilde{\mathcal{K}}^{-1}$ acts as an (retarded) time evolution operator, which evolves $\Pi$ from $t$ to $t+\gamma^{-1}$.

(3) The derivative expansion of $\Pi_{l}$, incorporating at most second-order derivatives, i.e., $\left\langle\Pi_{l}\right\rangle \rightarrow 2 d v_{X}+a^{2} \nabla^{2} v_{X}+$ $d(2 d-1) v_{X}^{2}+o\left[\nabla^{4} v,\left(\nabla^{2} v\right)^{2}\right]$.

Similar to the adiabatic elimination, the first two steps are justified on timescales $t>\gamma^{-1}$. It is the average timescale on which a coherently rotating spin is suddenly pushed back into the ground state by the emission of a photon and suddenly loses all its memory on the previous evolution. It is therefore precisely the autocorrelation time of a single spin and $\left\langle n_{l} n_{l}\right\rangle=\left\langle n_{l}\right\rangle(t)\left\langle n_{l}\right\rangle\left(t^{\prime}\right)$ if $\left|t^{\prime}-t\right| \geqslant \gamma^{-1}$. This incoherent emission process also counteracts the buildup of coherence between two adjacent spins such that the connected correlation function $\left\langle n_{j} n_{l}\right\rangle_{c} \equiv\left\langle n_{j} n_{l}\right\rangle-\left\langle n_{j}\right\rangle\left\langle n_{l}\right\rangle$ is dominated by local, classical correlations. Performing the coarse-graining volume average (2.16), the connected correlation function yields a contribution $O\left(N_{x}^{-1}\right)$ compared to the $O(1)$ product of expectation values and is negligible for $N_{x} \gg 1$ [78].

The third approximation, the derivative expansion, is $a$ priori not justified for first-order phase transitions since the relevant field fluctuations are strongly varying domain walls rather than extended spin waves at low momentum (i.e., small curvature). This may lead to an increased relevance of higher order derivative terms in the Langevin equation. Keeping track on the evolution of certain derivative terms during the renormalization group flow, however, shows that they have no considerable impact and can be neglected. This justifies the derivative expansion a posteriori.

The coarse-graining procedure yields the fundamental Langevin equation of our approach (prime denotes derivative with respect to the argument)

$$
\partial_{t} v_{X}=D \nabla^{2} v_{X}-V^{\prime}\left(v_{X}\right)+\xi_{X} .
$$

It describes the diffusive propagation $\left(D=\Omega^{2} a^{2} / \gamma\right)$ of $v_{X}$ in the potential

$$
V\left(v_{X}\right)=\frac{\Delta}{2} v_{X}^{2}+\frac{\mu}{3} v_{X}^{3}+\frac{\lambda}{4} v_{X}^{4},
$$


with $\Delta, \lambda>0>\mu$. The effective parameters in the Langevin equation are related to the master equation via

$$
\Delta=\gamma-\frac{\Omega^{2}}{2 \gamma}, \quad \lambda=\frac{4 \Omega^{2} d(2 d+1)}{\gamma}, \quad \mu=\frac{-2 \lambda d}{2 d+1} .
$$

The potential acquires a double-well structure for $|\mu|>$ $2 \sqrt{\lambda \Delta}$. This will always be fulfilled in the vicinity of the dark-state transition, see Fig. 3.

In addition, the density $v_{X}$ experiences the kinetic energy $D \nabla^{2} v_{X}$ and is subject to a nonthermal, multiplicative noise with kernel

$$
\left\langle\xi_{X} \xi_{Y}\right\rangle=\delta(X-Y) \gamma v_{X} .
$$

The noise acts locally on the field $v_{X}$ and, via Eq. (2.17), randomly kicks $v_{X}$ away from its current configuration. On top of the deterministic evolution, for each spatial coordinate $x$, the noise performs an independent random walk in time on $v_{x, t}$, i.e., $v_{x, t} \rightarrow v_{x, t+d t}=v_{x, t}+d v_{x, t}$. The expected step size $d v_{x, t}$ of the walk within one time step $t \rightarrow t+d t$ is $\left|d v_{x, t}\right|=\gamma v_{x, t}$. For smaller and smaller values of $v_{X}$ the random walk will on average need more and more time steps in order to leave a certain area. In particular for $v_{X}=0$ it becomes trapped and unable to escape. In the absence of any deterministic dynamics, the random walk will thus always terminate in the dark state [79].

In the case of the double-well potential $V$, the noise becomes crucial as the noise induced random walk is the only way to overcome the potential barrier. Since the step size of the walk is proportional to the density, it is much more likely to overcome the barrier when starting in a valley of large density compared to starting in a valley with small density. Without considering the effect of spatial fluctuations, the noise always prefers to stay in the valley of smallest density.

In the following we express all dimensionful quantities in units of the spreading energy $\omega \equiv D / a^{2}=\Omega^{2} / \gamma$ where appropriate, i.e., $\bar{\Delta} \equiv \Delta / \omega, \bar{\mu}=\mu / \omega$, and $\bar{\lambda}=\lambda / \omega$.

\section{Relation to Rydberg lattice models and optical bistability}

Driven dissipative first-order phase transitions have recently gained considerable experimental and theoretical attention not only in the context of driven Rydberg ensembles but also for nonlinear photon lattices [7], semiconductor microcavities [9], and lattice cavity QED with cold atoms [8,12]. Common to all of these platforms is (i) a nonlinear relaxation dynamics towards a flux equilibrium steady state that balances drive and dissipation and (ii) a regime of bistability (or even multistability) between two (or more) clearly distinguishable thermodynamic phases, each represented by a different steady state. The local dissipation, unavoidable due to photon loss and/or spontaneous emission of excited levels, counteracts the build up of long range coherence and sets a generic timescale $t_{\text {dis }}$ after which $\left(t>t_{\text {dis }}\right)$ the dynamics at the transition, though nontrivial, can be understood in terms of classical processes.

Though optical bistability as well as weakly detuned, driven Rydberg ensembles differ on the microscopic level it has been realized that in the bistable regime their dynamics can be well described by a classical Ising model in a magnetic
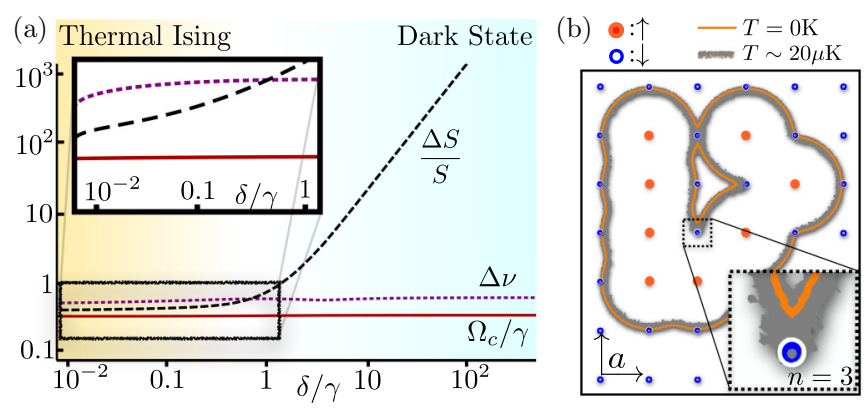

FIG. 2. (a) Discontinuity $\Delta v$, relative entropy increase $\frac{\Delta S}{S}$, and critical drive strength $\Omega_{c}$ as a function of the detuning $\delta$ (in units of $\gamma$ ) as predicted from mean field. The regime of small detuning $\delta / \gamma \geqslant$ 1 hosts the dissipative first-order Ising transition as well as common optical bistability $[7-9,21,73]$ while the regime of the present model with large detuning $\delta / \gamma \gg 1$ is defined by a strong relative increase of the entropy and thus far from a thermal phase transition. On the mean-field level both the coupling strength $\Omega_{c}$ and the jump $\Delta v$ are hardly affected by the detuning; the role of fluctuations, however, becomes increasingly pronounced as $\Delta S / S \rightarrow \infty$ in the dark-state regime. (b) Antiblockade area for Rydberg atoms at zero temperature (thin orange line) and finite temperature $T=20 \mu \mathrm{K}$ (thick gray line) in a lattice with depth $\sim 1 \mathrm{mK}$. The projection operators $\Pi_{l}$ are well realized with up to $n=3$ excited neighbors for $T=20 \mu \mathrm{K}$ (inset), while for $T=0 \mathrm{~K}$ only $n=1$ is realized.

field $[8,21,23,24,80]$. It is described by a Langevin equation (2.17) with a potential $V \rightarrow V_{\mathrm{IM}}$, which reads as

$$
V_{\mathrm{IM}}\left(v_{X}\right)=h v_{X}+\frac{\tilde{\Delta}}{2} v_{X}^{2}+\frac{\lambda}{4} v_{X}^{4},
$$

where the parameter $\tilde{\Delta}$ is different from $\Delta$ in Eq. (2.18), and $h$ acts as a magnetic field. In the case of optical bistability $h \sim \kappa$, where $\kappa$ is the photon loss rate [8,9], while for weakly detuned Rydberg atoms $h=-\frac{\gamma \Omega^{2}}{2 \gamma^{2}+\delta^{2}}$ is the off-resonant excitation rate.

The potential $V$ (2.18) can be brought into the form of $V_{\text {IM }}(2.21)$ via a constant shift $v_{X} \rightarrow v_{X}-\frac{\mu}{3 \lambda}$. It demonstrates that the deterministic dynamics of common, driven dissipative optical bistability, weakly detuned Rydberg ensembles, and the present model can be mapped onto each other. The noise kernel $\chi$, however, is crucially different. In the presence of a magnetic field, it is $\chi=|h|+\gamma v_{X}$ due to the Einstein relations. This sets a minimal fluctuation level $\chi_{\min }=|h|$, which destroys the dark state and generates a nonzero entropy for both bistable solutions. The relative entropy jumps at the transition $\Delta S / S \sim \gamma \Delta v_{X} /|h|$ (extracted from mean field and valid up to logarithmic corrections) become small and the transitions between the two states establish detailed balance, i.e., thermal equilibrium. In our model, however, detailed balance is absent in the bistable regime and cannot emerge at large scales due to the existence of the dark state. The relative entropy difference between the two metastable states diverges and noise activation becomes unidirectional, i.e., it is only possible from the finite density state to the dark state. This is the crucial difference between the present model and optical bistability, as well as previously discussed Rydberg models, see Fig. 2(a). 
In order to realize the nonequilibrium dynamics with the lattice models (2.1) and (2.3), it is essential to implement the projection operators $\Pi_{l}$ via the antiblockade mechanism. For a strict lattice model in which each Rydberg atom is exactly pinned at one lattice site, separated from each other by the lattice constant $a$, this is hardly possible. A single excited atom can easily facilitate the transition from the ground to the excited state of neighboring atoms via a precisely tuned antiblockade condition. If, however, two excited atoms are found in the neighborhood of a given ground state atom, the sum of their interaction energies shifts the effective excitation energy of the atom out of resonance, see Fig. 2(b) (orange line). This turns the antiblockade condition into an effective blockade condition and counteracts any facilitated excitation. In experimental realizations of Rydberg lattices, however, the motional degrees of the atoms experience a finite temperature of the order of several micro-Kelvin, which does not influence the spin degrees of freedom $[59,60,73]$. The atomic motion leads to temporal fluctuations of the van der Waals interaction strength between adjacent lattice sites of the order of 50\%$80 \%$ [59,60,73], which enables a much larger antiblockade area compared to strictly pinned atomic positions. For temperatures $T \approx 20 \mu \mathrm{K}$ in a lattice trapping potential with depth $\sim 1 \mathrm{mK}$ as used in Ref. [60], the antiblockade area averaged over several periods $t>1 / k_{\mathrm{B}} T$ is shown in Fig. 2(b). At this temperature $\Pi_{l}$ is effectively implemented for $n=1,2,3$ nearest neighbors and can be increased to $n \geqslant 4$ for larger temperatures $T \sim 100 \mu \mathrm{K}$ in the motional degrees of freedom. At the dark-state transition, we find $\left\langle v_{X}\right\rangle \leqslant 1 / 2$, i.e., in $d$ dimensions each atom has on average $2 d\left\langle v_{X}\right\rangle \leqslant d$ excited neighbors, which is covered by Fig. 2(b).

Balancing the antiblockade condition and the thermal atomic motion realizes the projection operators $\Pi_{l}$ for relevant densities at the dark-state transition. Better results can be obtained in free space, where the antiblockade radius is more flexible and becomes density dependent. The lattice spacing $a$ is then replaced by a flexible antiblockade radius $a_{R}(r, n)$, which depends on the number of excited atoms $n$ in the vicinity of the coordinate $r$. Another promising approach is the realization of the antiblockade condition via electromagnetically induced transparency (EIT) [81,82], with which one might be able to implement the projection operators exactly with the help of additional interatomic states (in progress). The present scheme is sufficient for low excitation densities, as found at the transition.

As a final remark, we want to mention that a Langevin equation in the form of Eq. (2.17) can be derived for the study of the dynamics of spatially separated, fragmented populations and their migration $[83,84]$. In these models the dark state corresponds to the extinction of all individuals of the population, while the spatially separated populations correspond to spatially well-separated droplets in our model. This connection is fascinating and opens up a different viewpoint on the Langevin equation in terms of population dynamics. It does, however, not reach beyond the mean-field limit.

\section{SCOPE AND METHODS}

The Langevin equation (2.17) encodes the complete physical content of the dark-state transition. Its (numerical) solution is, however, only rarely a viable approach to resolving the full dynamics of the model. Aside from computational cost in higher dimensions, the numerical realization of a multiplicative noise itself is a challenging and so far not unambiguously solved problem [85-89]. Therefore, we rely on a more diverse assortment of methods to develop an understanding of the phenomenology.

In order to familiarize the reader with the model, we start with a brief discussion of the corresponding nonequilibrium path integral in the saddle-point approximation. This repeats basic elements from the analysis in Refs. [5,29] and serves as an introduction to the phase diagram and to the notion of a first-order dark-state transition. It also gives rise to a number of questions to be addressed in this work.

We then develop a functional renormalization group (fRG) framework that can be applied in arbitrary dimensions and includes spatial fluctuations and noise beyond the mean-field picture. The approximate nature of this approach calls for cross validation, which we perform with the help of Langevin simulations in one dimension.

\section{A. Path integral formulation and mean-field phase diagram}

Any dynamics described in terms of a stochastic Langevin equation can be mapped onto a corresponding nonequilibrium path integral via the Martin-Siggia-Rose-Janssen-de Dominicis (MSRJD) construction [90-92]. This allows us to approach the theory via suitable functional methods such as saddle-point equations and the renormalization group. The MSRJD construction formally computes the partition function by summing over all stochastic trajectories (see, e.g., [93] for details). Introducing the purely imaginary, so-called response field $\tilde{v}_{X}$ it reads

$$
\mathcal{Z}=\int \mathcal{D}[v, \tilde{v}] \exp (-S[v, \tilde{v}])
$$

With the corresponding action

$$
S[v, \tilde{v}]=\int_{X} \tilde{v}_{X}\left[\partial_{t} v_{X}-D \nabla^{2} v_{X}+V^{\prime}\left(v_{X}\right)-\gamma v_{X} \tilde{v}_{X}\right] .
$$

It is at most quadratic in the response fields $\tilde{v}_{X}$, which is a consequence of the ferromagnetic dark state. This state displays no quantum fluctuations in the order parameter $n_{l}$ such that higher order noise terms, familiar from the Keldysh quantum path integral [16,93], become subleading in the limit $v_{X} \rightarrow 0$. Within our analysis, we validated that even at nonzero $v_{X}$ these terms do not qualitatively alter the picture obtained with the renormalization group and thus can be neglected right from the start.

We will now briefly review the saddle-point analysis of [5]. In the absence of spatial and temporal fluctuations $\left(v_{X}=v\right)$ one finds the saddle-point equations

$$
\begin{gathered}
\frac{\delta S}{\delta v_{X}} \stackrel{!}{=} 0 \Leftrightarrow \tilde{v} V^{\prime \prime}(\nu)-\gamma \tilde{v}^{2}=0, \\
\frac{\delta S}{\delta \tilde{v}_{X}} \stackrel{!}{=} 0 \Leftrightarrow V^{\prime}(v)-2 \gamma \tilde{\nu} v=0 .
\end{gathered}
$$

The stationary field configurations are considered to be insensitive to the noise level $(\tilde{v}=0)$ and the phase diagram is 


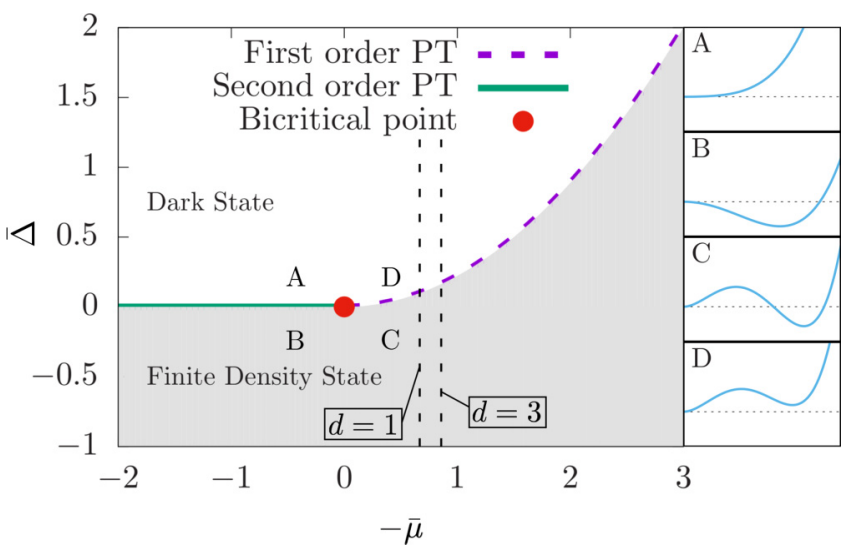

FIG. 3. Mean-field phase diagram for the action (3.2) at $\bar{\lambda}=1$. The finite-density phase is indicated by shading. A bicritical point at $\bar{\mu}=0$ separates regimes of first- (dashed line) and second-order (solid line) phase transitions. The shape of the potential $V(v)$ is sketched for the different regimes to the right of the plot. The black dotted lines indicate where the physical model (2.3) crosses the phase boundary in dimensions $d=1$ and $d=3$ (in the Rydberg model, the dark state is found for $\Omega \ll \gamma$ and the transition appears at $\Omega \approx \gamma$ ).

derived from the deterministic part of the action only:

$$
0 \stackrel{!}{=} V^{\prime}(v)=\Delta v+\mu v^{2}+\lambda v^{3} .
$$

Keeping $\lambda>0$ fixed, the phase diagram in Fig. 3 ensues. In general, the deterministic potential $V$ admits two qualitatively different states: a finite-density (fluctuating) state at $v_{f}=$ $\frac{\sqrt{\mu^{2}-4 \Delta \lambda}-\mu}{2 \lambda}$ and the (fluctuationless) dark state at $v_{d}=0$. For $\Delta<0, v_{f}$ is the only minimum of $V$, whereas for $\Delta>0, \mu<$ 0 both are present. Consequently, the transition between a fluctuating state with nonzero noise level and a fluctuationless phase with vanishing noise level as a function $\Delta$ is of second order for $\mu \geqslant 0$, whereas a first-order transition occurs for $\mu<0$.

The regime displaying a second-order phase transition, including nonequilibrium universality and scaling, was investigated in detail in Ref. [5]. Here we focus on the domain of first-order phase transitions $(\Delta>0, \mu>0)$. As opposed to second-order transitions, there is no universality associated with this type of phase boundary. Consequently, a different set of questions poses itself and shall be answered during the course of this work:

(1) Can the notion of a first-order dark-state transition persist beyond the saddle-point approximation and if so, how sensitive is it with respect to spatial dimensionality?

(2) What distinguishes a first-order dark-state transition from its thermal counterpart?

(3) Is there a similar notion of phase coexistence for dark states?

(4) Can we shed light on the dynamics at multiple scales by adapting (functional) renormalization group techniques?

\section{B. Renormalization group I: Full potential approximation}

At this point, it is not clear whether the mean-field phase diagram in Fig. 3 is at all a faithful representation of the system's physical behavior. The multiplicative noise, being the signature feature of the model, has been omitted completely so far. In previous work, this has partially been remedied by employing an optimal path approximation [5]. However, this approach does not account for corrections to the noise itself due to the interplay of deterministic dynamics and fluctuations. As we will point out later, such corrections can in fact be very sizable. Here we therefore develop an fRG scheme capable of implementing precisely this.

In previous work [5,49,55,94-96] (functional) RG techniques have successfully been applied to investigate critical phenomena in the presence of a dark state. Their unique advantage is a systematic inclusion of fluctuation effects beyond the noiseless mean-field or optimal path [93] approximations while being rather inexpensive compared to full-scale numerical simulations. In the following we will therefore extend the fRG scheme from [5] in order to accommodate an investigation of discontinuous phase transitions. We expect the nonperturbative fRG to be particularly well suited for this problem, as perturbative schemes are inherently unable to resolve the strong, nonlinear fluctuations between the two nontrivial minima of the deterministic potential which are ultimately expected to drive the phase transition.

The main objective of a functional renormalization group analysis is the computation of the effective action $\Gamma$, i.e., the generating functional of one particle irreducible correlation and response functions. This is achieved by solving the Wetterich equation [47]

$$
\partial_{k} \Gamma_{k}=\frac{1}{2} \operatorname{Tr}\left[\left(\partial_{k} R_{k}\right)\left(\Gamma_{k}^{(2)}+R_{k}\right)^{-1}\right] .
$$

Here $\Gamma_{k}$ is the so-called effective average action, interpolating between the microscopic $S=\Gamma_{k \rightarrow \Lambda}$ and the full effective $\Gamma=\Gamma_{k=0}$ actions. This is achieved by means of an (additive) regulator function $R_{k}$ that introduces a dependence on a scale parameter $k \in[0, \Lambda]$, with $\Lambda$ the overall energy or momentum cutoff.

While Eq. (3.5) by itself is an exact representation of the underlying field theory, its solution for nontrivial systems generally requires approximations. As $\Gamma_{k}$ does in principle facilitate all terms compatible with the symmetries of the respective system, approximations are invoked by reducing the number of terms kept during the calculation down to a manageable size. Thus, a truncated ansatz for $\Gamma_{k}$ is constructed, whose second functional derivative $\Gamma_{k}^{(2)}$ may then be used to compute the right-hand side of Eq. (3.5) and determine the evolution of the action.

The crucial point in obtaining reliable and accurate results is of course the very choice of terms to be kept in $\Gamma_{k}$. While canonically relevant or marginal operators are an obvious choice for weakly coupled systems (i.e., in the vicinity of a Gaussian fixed point), the situation is less clear if one is interested in the physics of interacting fixed points or even nonuniversal regimes. The latter case is of particular interest for this work, as it encompasses discontinuous phase transitions. Here concepts like canonical scaling or critical dimensionality do not apply and thus largely invalidate powercounting arguments.

Unfortunately, it has so far not been possible to devise a construction scheme for truncated $\Gamma_{k}$ that guarantees a steady improvement of results upon inclusion of further 
contributions. We therefore resort to the well-established derivative expansion [48] and benchmark our results against other methods whenever possible.

The generic ansatz for $\Gamma_{k}$ based on the action (3.2) at lowest nontrivial order in the derivative expansion is

$$
\Gamma_{k}=\int_{X} \tilde{v}_{X}\left[Z_{k} \partial_{t}+D_{k} \nabla^{2}\right] v_{X}+\int_{X} W\left(\tilde{v}_{X}, v_{X}\right) .
$$

Here $W_{k}\left(\tilde{v}_{x}, v_{X}\right)$ is the local potential that contains arbitrary (symmetry compatible) powers of $\tilde{v}_{X}, v_{X}$ and products thereof. Its initial value according to Eq. (3.2) is given by

$$
W_{\Lambda}\left(\tilde{v}_{X}, v_{X}\right)=\tilde{v}_{X}\left[\Delta v_{X}+\mu v_{X}^{2}+\lambda v_{X}^{3}-\gamma v_{X} \tilde{v}_{X}\right] .
$$

While it is straightforward algebra to determine the flow equation for $W\left(\tilde{v}_{X}, v_{X}\right)$, its actual evaluation cannot be done analytically anymore. Even numerically, it is a rather costly task, as it requires the discretization of $v_{X}$ and $\tilde{v}_{X}$ on a sufficiently fine-grained two-dimensional grid.

Considering simplifications, the different canonical scaling and thus relevance of field monomials $\tilde{v}_{X}^{m} v_{X}^{n}$ seems to justify a representation of $W_{\Lambda}\left(\tilde{v}_{X}, v_{X}\right)$ as a polynomial. However, this is not feasible in our situation since we are in a nonuniversal regime, where power-counting arguments are generally not valid. This manifests in two ways:

First, the deterministic potential in the vicinity of a discontinuous phase transition exhibits two competing minima even at mean-field level. Due to their finite separation in field space, a polynomial expansion of $V_{k}\left(v_{X}\right)$ about one minimum therefore becomes a rather bad approximation in the vicinity of the other one. Since the difference of the minima' values is crucial for determining the phase boundary, a low-order polynomial expansion will not yield accurate results [97,98].

Second, it can be shown [48] that the $V_{k}\left(v_{X}\right)$ is rendered convex for $k \rightarrow 0$. For the effective potential at a first-order phase transition, this amounts to a Maxwell construction. The ensuing straight line is represented at best asymptotically by a polynomial expansion even at intermediate scales. In addition, we demonstrate that the initial linear multiplicative noise is modified drastically and cannot be represented by a simple expansion.

Even if the deterministic potential is smoothened under the $\mathrm{RG}$ evolution such as our result in $1 \mathrm{D}$ indicate (see Sec. IV below), nonpolynomial features originating from its initial shape are still generated at intermediate scales.

We therefore refrain from a polynomial representation in both field variables and keep the full $v_{X}$ dependence on the deterministic potential as well as the noise kernel:

$$
W_{k}\left(\tilde{v}_{X}, v_{X}\right)=\tilde{v}_{X}\left[u_{k}\left(v_{X}\right)+\chi_{k}\left(v_{X}\right) \tilde{v}_{X}\right]
$$

with $u_{k}\left(v_{X}\right) \equiv V_{k}^{\prime}\left(v_{X}\right)$ and the initial conditions indicated in Eq. (3.7) above. We checked numerically that higher order terms $\sim \tilde{v}_{X}^{3}$ as naturally present in a Keldysh framework do not contribute qualitatively and give only small corrections to quantitative results (see below). On similar grounds, we set the wave function renormalization parameters $Z_{k}=D_{k}=1$ from now on and ignore higher derivative terms. The ansatz (3.8) therefore represents the most simple yet sufficient truncation to faithfully capture the phenomenology of first-order darkstate phase transitions.
The flow equations for the (derivative of the) deterministic potential and the noise kernel can now be computed:

$$
\begin{aligned}
\partial_{k} u_{k}= & -\frac{k^{d+1} v_{d} \chi_{k} u_{k}^{\prime \prime}}{d\left(k^{2}+{u_{k}^{\prime}}^{2}\right)^{2}} \\
\partial_{k} \chi_{k}= & -\frac{k^{d+1} v_{d} \chi_{k}}{2 d\left(k^{2}+{u_{k}^{\prime}}^{2}\right)^{4}}\left[3 \chi_{k} u_{k}^{\prime \prime 2}-8\left(k^{2}+u_{k}^{\prime 2}\right) \chi_{k}^{\prime} u_{k}^{\prime \prime}\right. \\
& \left.+2\left(k^{2}+{u_{k}^{\prime}}^{2}\right)^{2} \chi_{k}^{\prime \prime}\right]
\end{aligned}
$$

Here $v_{d}=\left[2^{d-1} \pi^{\frac{d}{2}} \Gamma\left(\frac{d}{2}\right)\right]^{-1}$ is the (normalized) volume of the $(d-1)$-dimensional unit sphere.

\section{Renormalization group II: Benchmarking}

For (nonequilibrium) systems with mean-field first-order phase transitions, there is neither literature (cf. [99-101] for equilibrium approaches) nor a stringent analytical argument for the sufficiency of the truncation scheme presented above. We therefore need to benchmark the results of our fRG computations in order to build confidence in the reliability of its predictions. In order to achieve this, we conducted numerical simulations of the Langevin equation (2.17) in one spatial dimension. In the following, we will present two different physical setups and compare the results of these simulations to the respective outcomes of our fRG approach.

\section{Thermal equilibrium}

A simple test case is provided by the thermal equilibrium limit. Since we consider the one-dimensional case with shortrange interactions, no phase transitions can be expected due to the exponential growth of entropy with number of domain walls [102]. Even if mean-field predicts the existence of first-order transitions, we expect the occurrence of a smooth crossover upon inclusion of fluctuations.

For an appropriate implementation in the Langevin equation (2.17), the noise correlation (2.20) has to be replaced by

$$
\left\langle\xi_{X} \xi_{Y}\right\rangle=2 T \delta(X-Y),
$$

with $T$ being the temperature. Our simulations were performed on a 1000 site lattice with periodic boundary conditions and for time steps of $\Delta t=10^{-1} \cdots 10^{-2}$ in units of the lattice spacing. We picked a regime that encloses a first-order phase transition at mean field, given by $\Delta=1, \lambda=1$ at $T=$ 0.25 . The outcomes for the average density $v_{X}$ as a function of $\mu$ are shown in Fig. 4 alongside the corresponding mean-field and $\mathrm{fRG}$ results. For the functional RG calculation, we set

$$
\chi_{\Lambda}\left(v_{X}\right)=\chi_{k}\left(v_{X}\right)=T,
$$

in order to implement detailed balance. Thus, our truncation is simplified further by not allowing for any feedback of the deterministically induced fluctuations into the flow of the noise kernel. This approximation can only be expected to be appropriate at sufficiently high temperatures. Since $\partial_{k} u_{k} \sim T$, it is obvious that the flow would be switched off for $T \rightarrow 0$. This situation therefore corresponds to a purely classical setup, where only thermal fluctuations are taken into account, whereas quantum fluctuations are neglected. Judging by the 


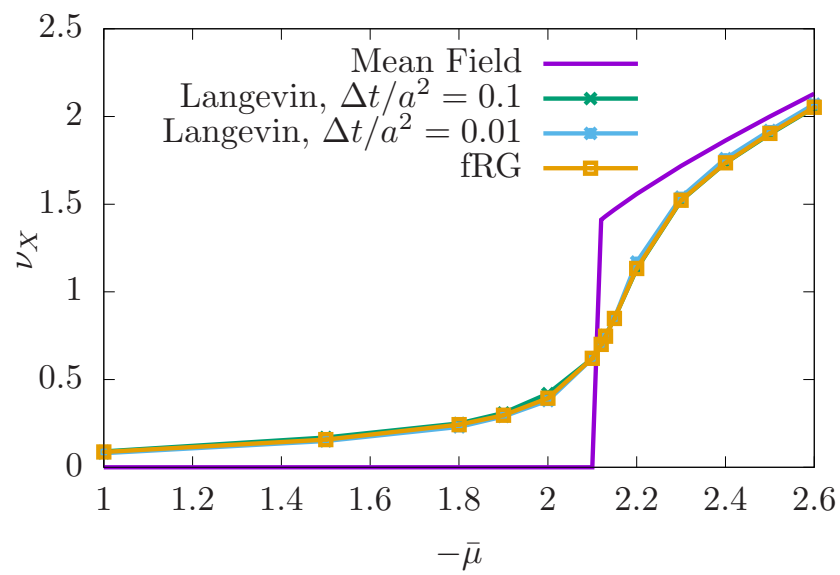

FIG. 4. Density $v_{X}$ as a function of $\mu$ for $\bar{\Delta}=1, \bar{\lambda}=1$ at $T / \omega=$ 0.25 . Langevin and fRG results agree quantitatively, whereas sizable corrections to the mean-field prediction are made. Statistical error bars on the Langevin results are not visible on the scale of the plot.

results presented in Fig. 4, functional RG is very well able to reproduce this limit, as the curves for the Langevin simulation lie practically on top of the fRG result.

\section{Nonequilibrium: Dark state}

The thermal limit mainly constitutes a test for the deterministic sector of the model. Naturally we need to benchmark our $\mathrm{fRG}$ also in a nonequilibrium situation, i.e., in the presence of nontrivial noise. We therefore implemented the Langevin equation (2.17) with the original multiplicative noise (2.20) as well. Unfortunately, this is not as straightforward as in the thermal case. In particular, the concrete realization of the multiplicative noise in a time-discrete setting is still an open problem that has not been solved unambiguously [88,89]. One basic issue is that upon discretization, a multiplicative noise is able to induce negative values for the density where it is itself not well defined anymore [85-87]. While no negative density can be observed since the respective fluctuations disappear on average, the definition of the noise kernel for negative densities does have an impact on the actual value of observables.

We employ three different definitions of the noise kernel for negative $v_{X}$ :

$$
\begin{aligned}
\chi_{\text {flat }} & = \begin{cases}\gamma v_{X}, & v_{X} \geqslant 0, \\
0, & v_{X}<0,\end{cases} \\
\chi_{\text {abs }} & =\gamma\left|v_{X}\right|, \\
\chi_{\text {barr }} & = \begin{cases}\gamma v_{X}, & v_{X} \geqslant 0, \\
\infty, & v_{X}<0,\end{cases}
\end{aligned}
$$

While the resolution of the temporal lattice did not play a big role in thermal equilibrium (cf. Fig. 4), this is not true for the present setup anymore. An extrapolation to $\Delta t=0$ would therefore be necessary for a quantitative comparison with fRG results. Due to the increased numerical effort necessary to achieve reasonable accuracy, and since our main focus is not on Langevin simulations, we refrain from doing so. In Fig. 5 results for different $\Delta t$ and all three realizations of the noise

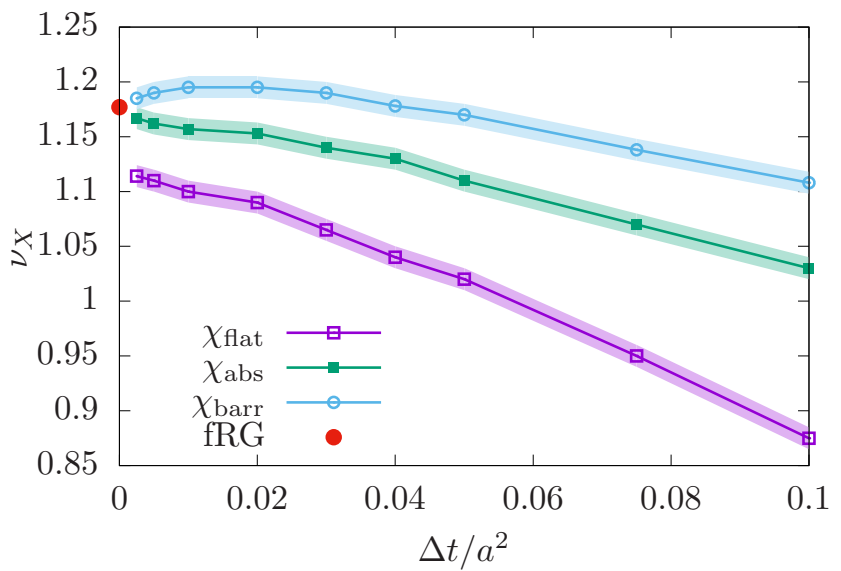

FIG. 5. Density $v_{X}$ at different temporal discretizations for constant $N_{t} \Delta t / a^{2}=100$ at $\bar{\Delta}=1, \bar{\mu}=-2.42, \bar{\lambda}=1$, and $\bar{\gamma}=0.5$. Statistical errors on the Langevin results are depicted by the shading around the data points. Approximate convergence towards the fRG results can be observed for dark-state realizations $\chi_{\text {abs }}$ and $\chi_{\text {barr }}$, cf. Eqs. (3.12).

kernel are presented in comparison with the fRG result. The configuration belonging to these data points is given by the (initial) values $\Delta=1, \mu=-2.42, \lambda=1$, and $\gamma=0.5$ on a grid of 100 sites. This setup lies well inside of a finite-density phase at mean field and also according to fRG results (see Fig. 7 below). For comparability, the number of steps times the temporal spacing $N_{t} \Delta t=100$ have been kept constant. Each single data point results from an average over 1200 sample densities. Statistical uncertainties are indicated by shading. While the estimate obtained with $\chi_{\text {flat }}$ is somewhat low, $\chi_{\text {abs }}$ as well as $\chi_{\text {barr }}$ are well compatible with the fRG result.

For completeness, it should be stated that convergence towards the continuous time and infinite volume limit is not achieved as easily in low-density regimes. While $N=100$ sites appeared to be sufficient for the setup presented in Fig. 5, this is not the case for smaller $|\mu|$ anymore. The reason is once again the difficulty of resolving the actual dark state in the Langevin simulations. Again, we refrained from a more sophisticated numerical analysis of the Langevin equation in this regime due to numerical cost. However, it can be stated that the position of tentative phase transitions appears to be compatible with the fRG result. The agreement is even better, if the flow equations (3.9a) re supplemented by a term $w_{k}\left(v_{X}\right) \tilde{v}_{X}^{3}$. No qualitatively notable differences were found, though, and the quantitative corrections are on the percent level. We therefore do not include $w_{k}\left(v_{X}\right)$ into our further analyses.

In conclusion, we found good agreement between numerical Langevin simulations and our functional RG results in and out of equilibrium. This gives us confidence that we can trust our fRG approach for an extended analysis of the one-dimensional as well as the three-dimensional cases.

\section{THE FATE OF THE DARK STATE IN ONE DIMENSION}

Including both spatial and temporal fluctuations beyond the mean-field approximation often has dramatic consequences on the stability of ordered phases and the nature of the corresponding phase transitions. This is most drastically expressed 
in one dimension, where in the presence of a finite noise level the entropy gain per excitation always wins against the energy cost [102] and no ordered phase can be stabilized (see Fig. 4). Spatial correlations in one dimension are so strongly pronounced that even infinitesimally small temporal fluctuations of the order parameter can grow rapidly in space and render the ordered state unstable. While the dark state itself does not experience any fluctuations and is thus immune to this destructive mechanism, any infinitesimal deviation from a pure dark state does experience strongly suppressed but finite fluctuations and it is thus a priori unclear whether a dark-state phase can persist against the generically strong fluctuation dynamics in one dimension.

\section{A. RG analysis}

Here we analyze the fate of the dark state in the presence of fluctuations. We apply the functional renormalization group approach introduced above, which includes temporal and spatial fluctuations on all energy and momentum scales, and numerical simulations of the corresponding Langevin equation. From the combination of both approaches, we obtain a comprehensive picture of the asymptotic dynamics in the regime where both the dark state and the fluctuating state are deterministically stable on the mean-field level.

From our analysis, we conclude that

(1) Fluctuations render one of the two mean-field steady states unstable and only one, unique steady state remains in the asymptotic time limit.

(2) The dark-state phase persists in the presence of spatial and temporal fluctuations and remains stable for intermediate and low potential barriers.

(3) For large potential barriers, the dark state becomes unstable and decays into a mixed state with nonvanishing field expectation value.
(4) Fluctuations enlarge the regime of the dark-state phase compared to the mean-field prediction but soften the predicted first-order phase transition towards a continuous, secondorder transition throughout the whole parameter regime.

The most striking result of these conclusions is that fluctuations are sufficiently strong to remove the mean-field predicted first-order phase transition completely and replace it by a continuous second-order phase transition throughout the entire one-dimensional parameter regime [100]. On the other hand, they are sufficiently weak that a dark-state phase, which is represented by only a single fluctuationless point in phase space, can be stabilized for an extended parameter regime. Similar behavior has been observed for classical, absorbing state phase transitions in one spatial dimension, for which first-order phase transitions induced by short ranged processes are conjectured not to exist [103], but second-order absorbing state phase transitions have been established [31,32].

The observed softening of the transition, which is a major feature of the one-dimensional dynamics, can be understood in a two-staged coarse-graining procedure. In order to do so, imagine one initializes the dynamics in the mixed phase but close to the phase border (e.g., configuration C in Fig. 3). In addition to noise induced, small oscillations around the deterministic field expectation value (green configuration in Fig. 1), some rare, strong noise kicks let the field climb up and eventually overcome the potential barrier between the mixed and the dark state. This leads to the formation of local clusters with exponentially small field configurations, see Fig. 6 . In one dimension, the interplay between noise and kinetic energy, however, leads to spatially extended, fluctuating interfaces between the dark-state clusters and the finite-density phase, which spoil the picture of well-defined domain wall interfaces (red configuration in Fig. 1). As a consequence, metastable saddle-point configurations, known as droplets or instantons, that interpolate smoothly between the two phases
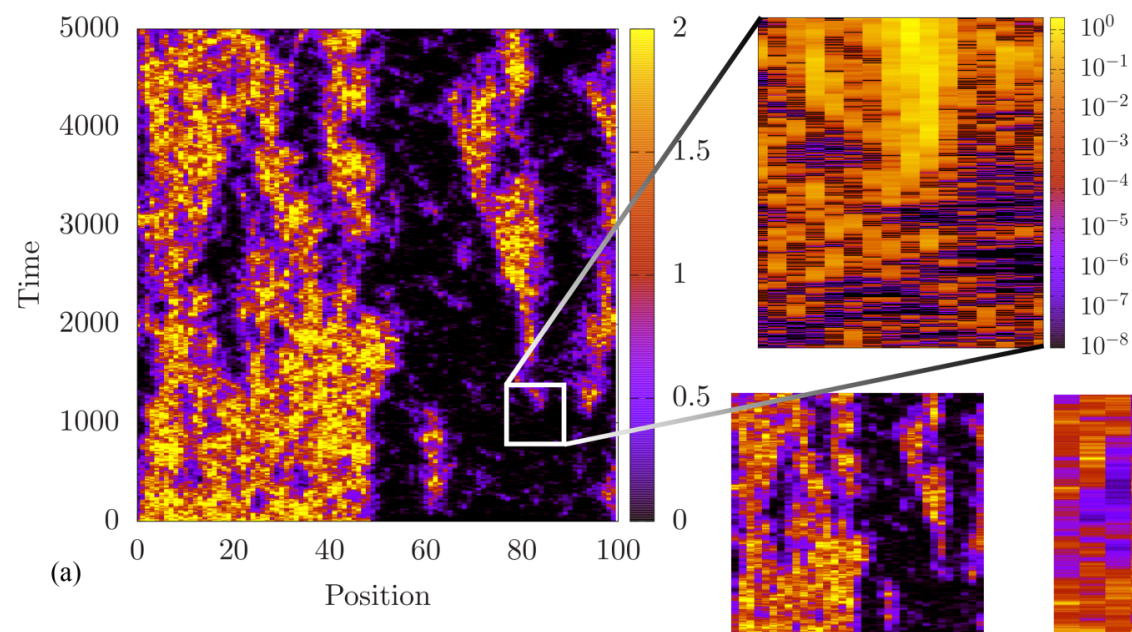

(b) (a) $-2$ 3 
(blue configuration in Fig. 1) are only observable on short times and become rather unstable at intermediate and larger times. This leads to a breakdown of the droplet picture in one dimension.

Upon coarse graining, the noisy interfaces between the two phases continuously smoothen the potential well separating the dark and the mixed state, which leads to a continuously varying density and, close to the phase transition, to the emergence of scale invariance on intermediate and large distances. This is observed in the numerical simulations of the Langevin equation, see Fig. 6, as well as in the numerical solution of the fRG flow equation. Within the latter, the potential barrier separating the dark and the mixed state vanishes smoothly while short distance modes are integrated out, see Figs. 8(a) and $8(b)$. Already on intermediate scales, the potential $V_{k}$ develops a single minimum and becomes convex, indicating the absence of sharp domain walls between dark and mixed states [57].

This observation, quantitatively and qualitatively, resembles the dynamics close to a second-order phase transition in the presence of a nonequilibrium noise. Indeed, increasing the noise strength, the Langevin simulations display a smoothly vanishing order parameter expectation value instead of the predicted, first-order jump. Within the fRG framework, the corresponding observation is that on intermediate scales the potential barrier fades away, leading to a single deterministic minimum and the breakdown of bistable behavior, see Figs. 8(a) and 8(b). Continuing coarse graining to the largest scales, integration over the long distance modes lets this minimum continuously move towards zero field expectation value, i.e., the dark state. At the transition, the minimum reaches $v=0$ at asymptotically small scales. In the RG interpretation, this describes the continuous divergence of the correlation length, i.e., the emergence of scale invariance as the transition is approached. The removal of the barrier during the flow with major corrections to the position of the nontrivial minimum still ahead also provides an intuitive explanation for the failure of the droplet picture: in a convex potential with only one minimum, no metastable droplet solution can be defined anymore. In the vicinity of the phase transition, the RG evolution always removes the potential barrier first and only then merges the nontrivial minimum with the dark state [57]. While dropletlike fluctuations therefore do play a role at short length scales, the fate of the minimum is ultimately decided by complex field configurations such as indicated by the red line in Fig. 1.

Another general consequence of the presence of fluctuations is the increased probability of the system to end up in the dark-state phase, despite being in an mixed state configuration on deterministic grounds alone. Thus, an increased initial noise level leads to an enlarged dark-state phase, see Fig. 7.

While we have discussed the reasons for the existence of a dark-state phase beyond mean field, one could also wonder about the stability of the finite-density phase in the presence of a dark state. Intuitively, there is always a possibility that some rare fluctuation drives the whole system into the zero-density dark state from which it cannot escape anymore. In fact, such events do plague Langevin simulations which are by construction bound to finite systems $[88,89]$. The infrared endpoint of the RG evolution, on the other hand, provides access to

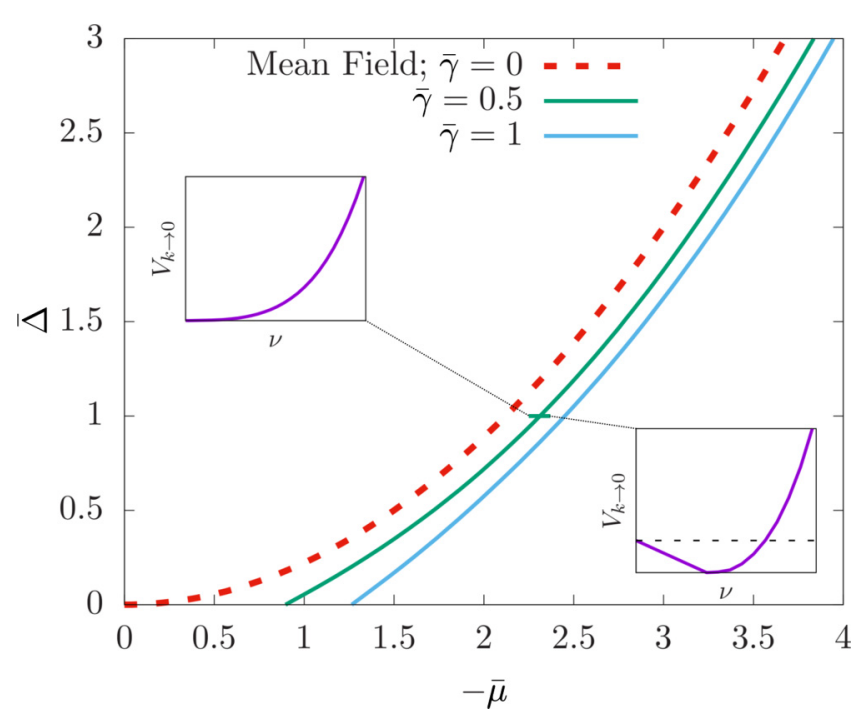

FIG. 7. Phase diagram of the one-dimensional system in the $\bar{\Delta}-\bar{\mu}$ plane for fixed $\bar{\lambda}=1$. The mean-field first-order transition (dashed line) is changed to second order (solid lines) upon inclusion of fluctuations. Increased initial noise levels lead to a larger dark-state phase. The insets show the asymptotic potential $V_{k \rightarrow 0}$ for the two different phases close to the transition for an initial noise level $\bar{\gamma}=0.5$. The complete evolution of the potential and noise vertex at these points can be found in Fig. 8 .

the steady state itself. While fluctuation effects are included which have to be accounted for by averaging over samples in a Langevin treatment, this state is not necessarily noiseless: the noise kernel $\chi_{k}$ (and potentially higher order operators as well) do evolve alongside the deterministic potential $V_{k}$. They generally remain finite for $k \rightarrow 0$, i.e., even after all fluctuations are integrated out. The existence of a nontrivial minimum in $V_{k \rightarrow 0}$ in itself is therefore not yet sufficient to argue for the stability of the finite-density phase, as the noise $\chi_{k}$ has to be taken into account also in the deep infrared.

Figure $8(\mathrm{c})$ shows the evolution of the noise kernel for a configuration inside the finite-density phase. The crucial finding is that for $k \rightarrow 0$, the initially single (pointlike) dark state at $v_{X}=0$ spreads into a continuum, i.e., to finite values of $v$. While it does not necessarily reach the value of the steady state $v_{f}$, it suppresses any noise between $v_{f}$ and the initial dark state at $v_{X}=0$. Since at the same time $u_{k \rightarrow 0}$ becomes convex and establishes a single minimum at $v_{f}$, the dark state becomes unstable and the coarse-grained Langevin equation describes deterministic, noiseless motion towards a mixed steady state.

The universality class at this second-order phase transition could not yet be determined. The computation times for a sufficiently clear resolution of the critical exponents in the limit $k \rightarrow 0$ are currently requiring too many resources and one possibly has to think about another approach to detect the critical exponents. We want to stress here that both the noise kernel $\chi_{k}$ and the potential $u_{k}$ become nonpolynomial functions, which prohibits a perturbative RG analysis of this second-order phase transition. We can rule out, however, that it falls into the directed percolation universality class since the characteristic rapidity inversion symmetry [31] is broken 


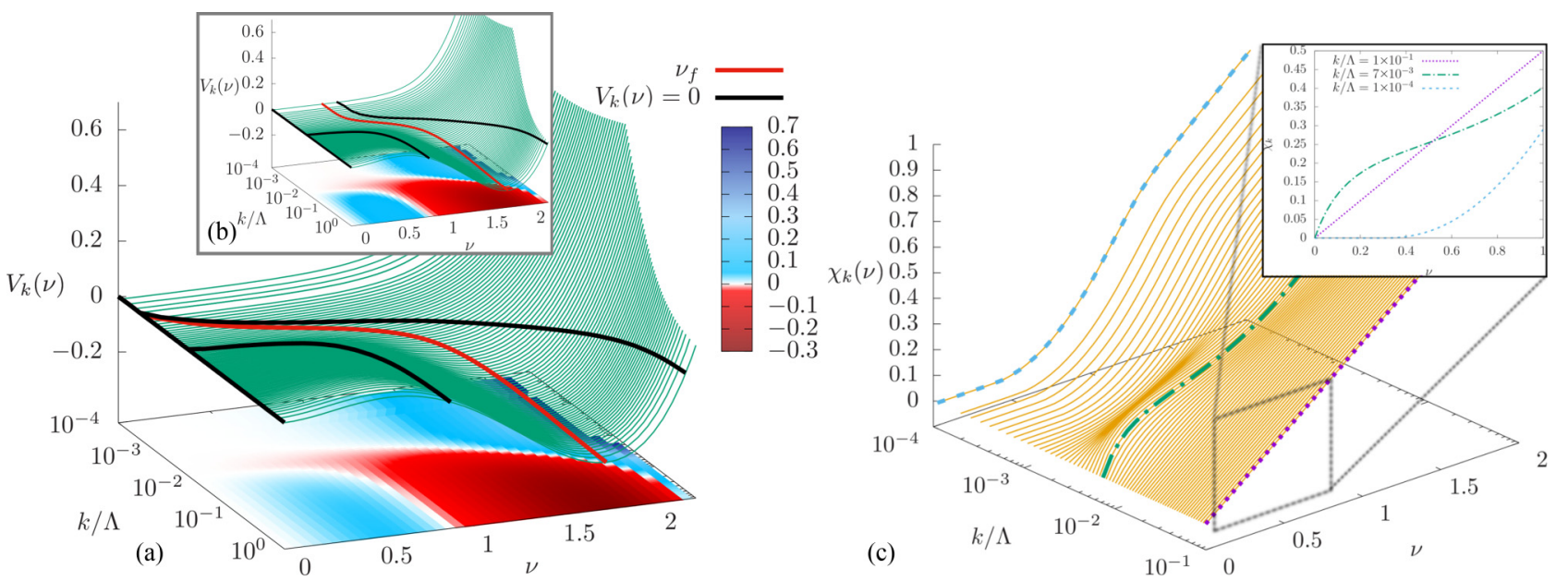

FIG. 8. Evolution of the deterministic potential $V_{k}$ and noise kernel $\chi_{k}$ for $\bar{\lambda}=1, \bar{\Delta}=1, \bar{\gamma}=0.5$, and (a) $\bar{\mu}=-2.33$, (b) and (c) $\bar{\mu}=$ -2.35 in $d=1$ dimensions. Before the dark state becomes the only nontrivial minimum at $k \rightarrow 0$, the potential barrier is removed [red and black solid lines in (a)]. Inside the finite-density phase, the nontrivial minimum persists for $k \rightarrow 0$ while $V_{k}$ is rendered convex at $k>0$ (b). At the same time, the dark state spreads in the noise kernel $\chi_{k}$, thus stabilizing the finite-density state [(c) and inset].

at any $k>0$ by a nonpolynomial potential. It has been shown that deviations from a second-order polynomial potential are RG relevant in $d=1$ [5] and thus will persist on the largest wavelengths, denying the restoration of rapidity inversion and the directed percolation universality at $k=0$.

\section{B. Experimental signatures}

The emergent second-order phase transition in low dimensions is, on the one hand, hard to characterize even with very advanced renormalization group schemes or (due to the multiplicative noise) with numerical methods. On the other hand, it denies any relation to known universality classes due to the absence of symmetries (e.g., no rapidity inversion, $\mathbb{Z}_{2}$ symmetry, or detailed balance) and without a well defined upper critical dimension $d_{c}$. This points towards a novel, so far uncharacterized universality class far from thermal equilibrium. The exploration of this universality class can be strongly supported by the analysis of the continuous evolution of the expected Rydberg state density $\left\langle v_{X}\right\rangle$ close to the transition. It enables the experimental detection of the transition's critical driving strength $\Omega_{c}$ and detuning $\delta_{c}$ and the measurement of basic critical exponents, including the order parameter exponent $\beta$, the conjugate field exponent $\sigma$, and the susceptibility exponent $\gamma$. They are defined via the response of $\left\langle v_{X}\right\rangle$ towards fluctuations in $\Omega$ and $\delta$, i.e.,

$$
\begin{gathered}
\left\langle v_{X}\right\rangle \sim\left|\Omega-\Omega_{c}\right|^{\beta}, \\
\left\langle v_{X}\right\rangle \sim\left|\delta-\delta_{c}\right|^{\beta / \sigma}, \\
\partial\left\langle v_{X}\right\rangle / \partial \delta \sim\left|\Omega-\Omega_{c}\right|^{-\gamma} .
\end{gathered}
$$

In the present setup, mean-field theory does not predict a second-order phase transition and the noise kernel for $k<\Lambda$ becomes nonpolynomial denying the derivation of a faithful effective model at intermediate scales. Consequently, no mean-field predictions for the critical exponents are obtained from theory. The experimental detection of some critical exponents would thus already be very helpful to embed this phase transition in the framework of nonequilibrium statistical mechanics.

In the numerical simulations we defined the cutoff momentum by setting $D \Lambda^{2}=2 \gamma$, which $\left[D=\Omega^{2} a^{2} / \gamma\right.$, see below Eq. (2.17)] implies $a=c / \Lambda$, where $c=\sqrt{2} \gamma / \Omega$ is a dimensionless prefactor, which is of order $O(1)$ in current experiments, and $a$ is again the lattice constant. The scaling dynamics of the potential and noise kernel set in at $k \approx 10^{-2} \Lambda$, see Fig. 8. The corresponding length scale $x_{k}=$ $k^{-1}=100 a / c$ indicates that renormalization effects beyond mean field will affect the dynamics in lattices of about 100 sites (or antiblockade radii). Both $V, \chi$ reach their asymptotic form for $k<10^{-3} \Lambda$, such that on system sizes $x_{k} \geqslant 10^{3} a / c$ universal scaling corrections beyond mean field are fully established. Current Rydberg lattice experiments aim towards the realization of $O(100)$ perfectly controllable lattice sites while Rydberg gases in harmonic trap geometries, by trading some of their controllability, already reach sizes of $O\left(10^{3}\right)$ blockade radii. This brings the exploration of an uncharacterized nonequilibrium critical point into reach of Rydberg experiments.

Combining these system sizes with the high tunability of driven dissipative Rydberg ensembles might even offer to directly monitor the transition from the microscopic, few atom physics to the many-body physics on macroscopic scales. As a function of the effective system size, controllable e.g., via the trap size, atomic density, and the detuning, experiments may go from rather small system sizes to larger and larger systems. By including more and more lattice sites, the experiment should thus be able to observe a transition from a few-body scale, $\sim O(10)$ sites, on which the mean-field bistability persists, to an intermediate scale $\sim O\left(10^{2}\right)$ sites, at which the bistable dynamics is strongly renormalized by long wavelength fluctuations, and to the many-body scale, $\sim O\left(10^{3}\right)$, at which the bistable regime is completely replaced by the discussed second-order phase transition. 


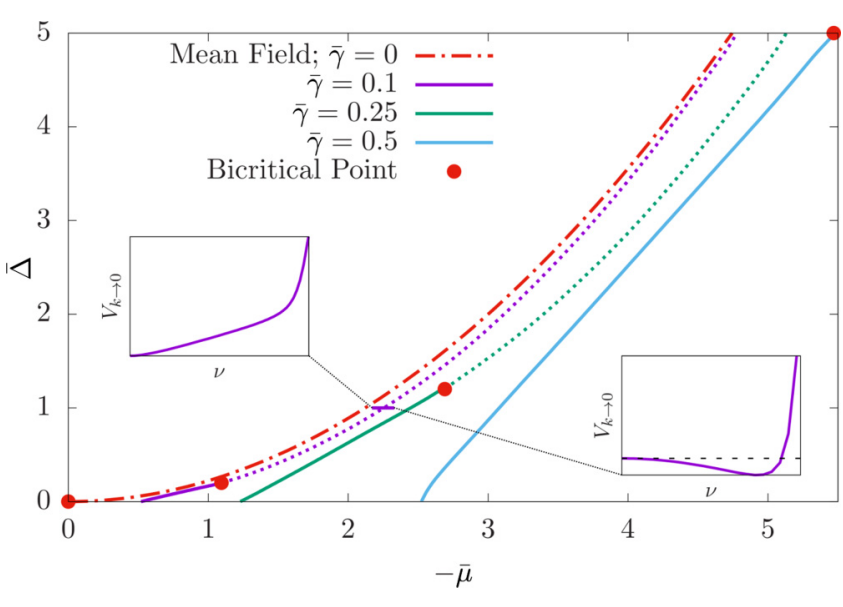

FIG. 9. Phase diagram of the three-dimensional system in the $\bar{\Delta}-\bar{\mu}$ plane for fixed $\bar{\lambda}=1$. The mean-field first-order transition (dot-dashed line) is gradually changed to second order (solid lines) upon increase of the initial noise level. First-order phase transitions are generally present beyond mean field (dotted lines), giving rise to a bicritical point at $\bar{\Delta} \neq 0$. Increased initial noise levels also lead to a larger dark-state phase. The asymptotic potential $V_{k \rightarrow 0}$ for the two different phases close to the transition is displayed in the two insets for an initial noise level $\bar{\gamma}=0.1$. The full evolution of the potential and noise vertex at these points is shown in Fig. 10.

\section{PERSISTENCE OF THE DISCONTINUOUS DARK-STATE TRANSITION IN HIGHER DIMENSIONS}

In higher dimensions, spatial fluctuations generally lead to less drastic modifications of the underlying mean-field picture. Only for very small potential barriers, the phase transition follows the same mechanism as in one dimension and becomes second order. We focus on intermediate to large potential wells, where we find that the first-order transition between the dark and the finite density state persists in the presence of noise and spatial fluctuations for $d \geqslant 2$ (see Fig. 9). For such initial potential barriers the double-well structure of the potential persists up to the largest wavelengths and $V_{k}$ becomes convex only in the limit $k \rightarrow 0$, establishing a single minimum. At the transition, the minimum jumps between $v_{X}=0$ and $v_{X}=v_{f}$ discontinuously such that the transition is of first order. This change in the potential evolution is accompanied by a drastic modification of the noise vertex flow compared to the case of a second-order transition and the emergence of a sharp momentum scale for the onset of the evolution. These observations match with the phenomenology of first-order phase transitions that are driven by nucleation and growth of droplets.

\section{A. Droplet phenomenology}

In order to discuss the results from the fRG approach, one needs a better understanding of the dynamics at a first-order phase transition and adopt the corresponding picture to the present nonequilibrium setting. We briefly review the conventional phenomenology and adapt it to the present setting.

In higher spatial dimensions, local field fluctuations are suppressed and typically modify the mean-field picture only on the quantitative level, i.e., the dynamics in higher dimensions is dominated by small fluctuations around the saddle points of the action. The homogeneous saddle-point solutions $v_{X}=0$ and $v_{X}=v_{f}$ in Eq. (3.4), and corresponding small fluctuations, do, however, not interpolate between the dark state and the finite density phase and one has to go beyond a homogeneous approach in order to describe the discontinuous transition.

In the common picture [1,2], first-order phase transitions are driven by the nucleation and the growth of droplets. Droplets are metastable field configurations which locally interpolate between the two different phases. To illustrate this, say the system is initialized in the finite density phase $v_{x, t=0} \approx v_{f}$ for all $x$. Tuning the system towards the transition by increasing the noise strength (or lowering the potential barrier) induces local transitions between the finite density solution $v_{X}=v_{f}$ and the zero density configuration $v_{X}=$ 0 . Since spatial fluctuations are strongly suppressed, these transitions will only pass an inhomogeneous saddle point

$$
0 \stackrel{!}{=} \frac{\delta S}{\delta \tilde{v}_{X}}=V^{\prime}\left(v_{X}\right)-D \nabla^{2} v_{X}
$$

which interpolates between the finite density phase and a noise induced excitation to the top of the potential barrier $v_{X}=v_{\max }$, see Fig. 1. For a droplet centered around $x=x_{0}$ this is expressed via the boundary conditions

$$
\begin{aligned}
& v_{X}=v_{f} \quad \text { for }|x| \rightarrow \infty, \\
& v_{X}=v_{\max } \quad \text { for } x=x_{0} .
\end{aligned}
$$

This configuration then deterministically reaches the dark state and nucleates a droplet.

Equation (5.1) requires local balance of kinetic and potential energy and the most relevant configurations are those which are most likely activated by the noise $\chi$. Since the present noise is field dependent, the structure of the most likely droplets will be a function of all the parameters, including the initial noise strengths. Their precise behavior cannot be determined quantitatively from a saddle-point equation alone. Qualitatively, however, its solutions may still be expected to convey valuable information. What all of them have in common is that they are smooth and form a sharp domain wall separating the dark-state configuration inside the droplet from the finite density region outside the droplet [104] (blue line in Fig. 1). This is in contrast to the strongly fluctuating field configurations (red line in Fig. 1) dominating the longwavelength dynamics in one dimension.

In this phenomenology, there exists a minimal extent of a droplet $\xi_{D}$, such that no droplet is formed below this size and thus no transitions between the minima occur on length scales $x<\xi_{D}$. While the value of $\xi_{D}$ depends in principle on all microscopic parameters and cannot be determined analytically, we observe the emergence of an extremely sharp, intermediate momentum scale $k_{D}$ during the RG flow. It marks the sudden onset of a fast evolution of both $V_{k}$ and $\chi_{k}$, see Fig. 10(d). Associating this sudden onset with the formation of the smallest possible droplet allows us to identify $k_{D} \approx \xi_{D}^{-1}$ within the limits of our approach [105]. Appositely, this scale is absent at the second-order transition. 


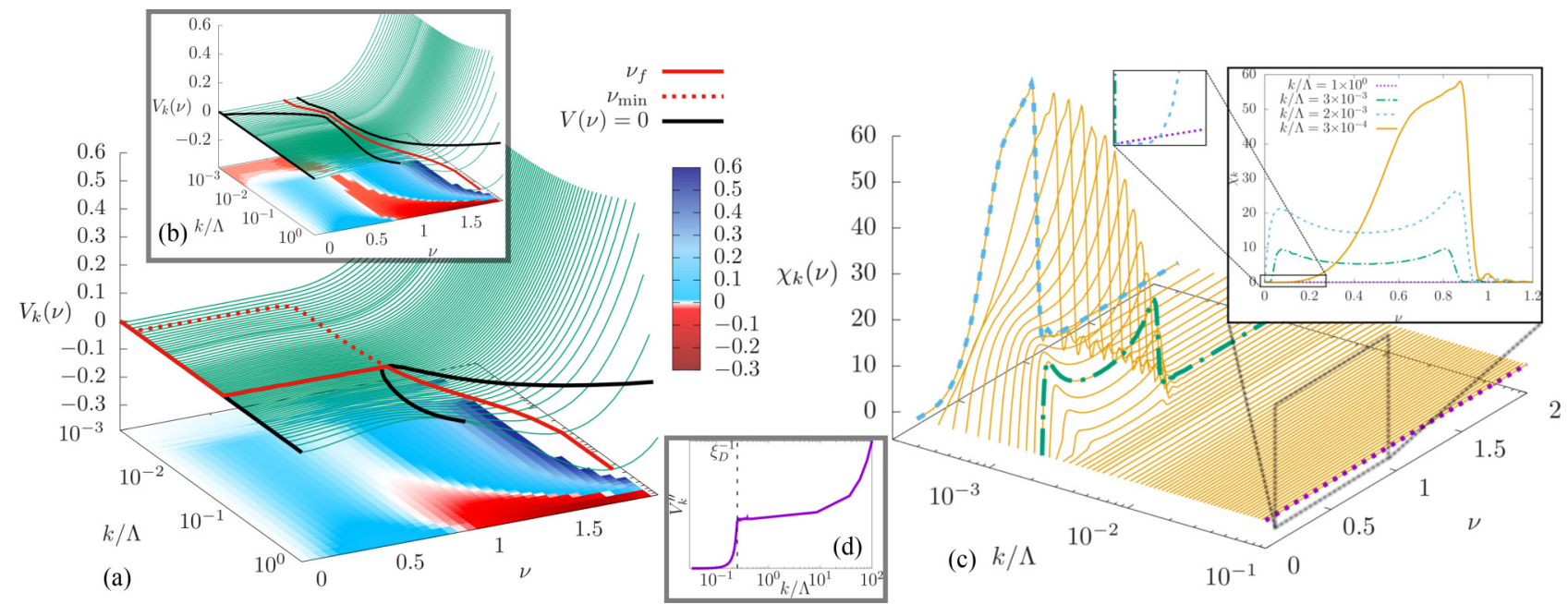

FIG. 10. Evolution of the deterministic potential $V_{k}$ and noise kernel $\chi_{k}$ for $\bar{\lambda}=1, \bar{\Delta}=1, \bar{\gamma}=0.1$, and (a) $\bar{\mu}=-2.32$, (b) and (c) $\bar{\mu}=-2.33$ in $d=3$ dimensions. The RG evolution decides first about the global minimum of the potential and only then renders the potential convex: the minimum at $v_{\min }$ becomes local at intermediate scales (a) and (b). The RG evolution of $V_{k}^{\prime \prime}(0)$ is shown in (d), providing a measure for the nonconvexity. The droplet scale $\xi_{D}^{-1}$ is indicated by a sudden sharp drop. Upon removal of the potential barrier, $\chi_{k}$ grows by three orders of magnitude and exhibits a bimodal structure (c). Only in the deep IR, fluctuations in the vicinity of the initial dark state are suppressed again. The insets provide an enhanced view on this event.

Once a droplet of the dark-state phase is formed, its evolution underlies the initial Langevin equation (2.17). For a large, homogenous droplet in dimensions $d>2$, this equation is dominated by the potential energy $V\left(v_{X}\right)$. It will grow or shrink with a deterministic velocity $v_{D}=-\Delta V=V\left(v_{f}\right)$, i.e., collapse for $V\left(v_{f}\right)<0$ and expand for $V\left(v_{f}\right)>0$. This picture neglects spatial fluctuations at the domain walls and noise. Within the fRG approach, however, noise and spatial fluctuations are integrated out and lead to the continuous renormalization of $V_{k}$ and $V_{k \rightarrow 0}\left(v_{f}\right)$ becomes the asymptotic droplet velocity.

We confirm this saddle-point picture of the transition in dimensions $d \geqslant 2$ even in the presence of spatial fluctuations and noise and identify indeed a first-order phase transition between the dark and the finite density phase for a large parameter regime. The transition is, however, in some aspects different from a first-order transition at (thermal) equilibrium, which we will briefly discuss and address qualitatively in the following:

(1) The nucleation probability of droplets in a dark-state region $v_{X} \approx 0$ is strongly suppressed by the small noise level $\sim v_{X}$ and vanishes completely for the dark state. In the absence of additional noise channels, an initial dark state always remains dark.

(2) The noise kernel prefers the dark state over the finite density state, i.e., there are much larger fluctuations inside than outside of a droplet. Noise and spatial fluctuations are therefore expected to modify $v_{D}$ and the phase boundary noticeably.

(3) The saddle-point basin of attraction for the dark state encompasses densities $0 \leqslant v_{X} \leqslant v_{\max }$. The noise in this regime is, by construction, generally suppressed. One might ask whether the corresponding, small nucleation rate can induce significant transitions to the finite density state and whether the extended basin of attraction for the dark state will persist in the presence of fluctuations or not.

\section{B. Droplets within the fRG framework}

The clear evidence for a first-order phase transition in the asymptotic fRG flow and the corresponding emergence of a sharp intermediate momentum scale for the onset of the flow strongly support the droplet interpretation of the first-order transition. Still, it is not immediately obvious if and how the peculiarities of spatially inhomogeneous droplet solutions are accounted for within our RG framework, since we always project onto spatially constant density profiles. However, this does by no means inhibit fluctuations which are integrated over to acquire an arbitrarily complicated spatial structure. While we thus do not have a direct, quantitative handle on verifying the droplet picture, we will discuss further distinctive features of the RG flow that match very well with this framework.

Let us first consider the flow of the deterministic potential for a configuration very close to a first-order phase transition, see Fig. 10. While the potential shows significant evolution on intermediate and large scales, the potential barrier is removed only after the RG evolution has decided about the global minimum of the potential [57], cf. Figs. 10(a) and 10(b). This is in contrast to a second-order transition, where longwavelength fluctuations account for considerable corrections or even destruction of the nontrivial minimum even after the barrier is removed [106]. This constitutes a strong hint on the nature of the fluctuations: as long as a potential barrier exists, droplets are well-defined excitations. In the vicinity of the first-order transition, these droplets therefore exist until the fate of the nontrivial minimum is decided in an RG sense and the transition is droplet driven.

However, these findings do not yet guarantee that these particular field configurations are in fact dominating the physics of the phase transition. In order to bring further clarification, we consider the flow of the noise kernel $\chi_{k}$ as well, see Fig. 10(c). First we note that the noise amplitude grows by almost three orders of magnitude within a comparatively small 
window of RG scales [77]. This happens precisely at the scale $k_{D} \approx \xi_{D}^{-1}$, discussed in the previous section, where the potential barrier is in the process of being removed, cf. Fig. 10(d). Second, as long as the flow has not reached the deep infrared, the noise kernel exhibits a very peculiar bimodal structure with the peaks roughly located at the positions of the nontrivial minima of the deterministic potential. This is exactly what one would expect for fluctuating droplet configurations, which suddenly jump from one minimum of the potential to the other, i.e., between the phases, and generate large field fluctuations around these minima.

Only when the regime of extremely long wavelengths is reached, droplets begin to subside and density fluctuations between the dark state and the finite density state become strongly suppressed, see Fig. 10(c). This eliminates the possibility of local transitions between zero density and finite density field configurations, which stabilizes both a thermodynamic finite density phase [107] and phase coexistence at the transition [see Fig. 10(d)].

This interpretation of the noise kernel is further strengthened when reconsidering the one-dimensional case. As argued in Sec. IV above, droplets are not expected to be the dominant fluctuations driving the phase transition. Indeed, the evolution of the noise kernel in Fig. 8(c) and also in the zero-density phase (not displayed here) does not display the bimodal behavior encountered in the vicinity of a first-order phase transition.

\section{Coexistence point}

Another aspect of the dark-state transition is the formation of generically expected phase coexistence at the first-order transition point, and the question whether a finite density on the one hand and the absence of fluctuations on the other can coexist.

Following the fRG analysis one finds that exactly at the transition both the deterministic force and the noise kernel vanish for an extended set of field configurations $0 \leqslant v_{X} \leqslant v_{c}$ (cf. Fig. 10). We now argue that this finding is generic to first-order dark-state phase transitions and neither an artifact of our theoretical approach nor a specific feature of this setup. For any initial noise and potential configuration, the effective action $\Gamma_{k=0}$ must be a convex function of the fields $v_{X}, \tilde{v}_{X}$. For the dark-state configuration both the noise kernel $\chi_{k}\left(v_{X}=\right.$ $0)=0$ and the potential $V_{k}\left(v_{X}=0\right)=0$ are, however, pinned to zero for all $k$. Requiring the coexistence of the dark and the finite density state at the transition, i.e., the absence of any deterministic or noise induced drift from one to the other, under the above conditions must therefore lead to a flat potential and vanishing noise kernel for an extended field configuration.

What we have to keep in mind, however, is that on the way from the master equation to the fRG results, the system has undergone a strong coarse-graining procedure. A vanishing noise kernel in this coarse-grained picture per se does not indicate that each individual spin is fluctuationless. Rather it shows the absence of fluctuations on thermodynamically large length scales. This specific feature of a first-order dark-state transition can be understood in the droplet picture. At the coexistence point, both phases are stable and one can thus

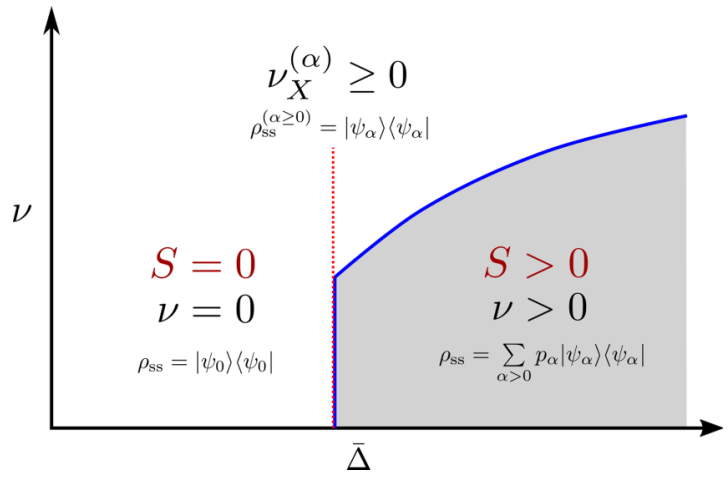

FIG. 11. Illustration of the first-order dark-state transition as a function of $\bar{\Delta}$ in the field theory and in the Langevin equation picture. The dark-state phase is characterized by a vanishing density field $v=0$ of spin-up atoms and a pure steady state $\rho_{\mathrm{SS}}=\left|\psi_{0}\right\rangle\left\langle\psi_{0}\right|$, Eq. (2.4). In the finite density phase, the steady state has finite density $v>0$ as well as nonvanishing entropy $S$ and noise level. Precisely at the transition, all field configurations $0 \leqslant v \leqslant v_{c}$ represent noiseless steady state solutions, which we interpret as the presence of an extensive number of dark-state configurations $v_{X}^{(\alpha)}$. Tentative analogies to fully quantum systems are indicated and discussed in the Outlook.

insert a thermodynamically large, stable droplet, which will not expand, nor move, nor shrink considerably. The only expected fluctuations happen at the domain wall of the droplet, which is a sparse region. The absence of fluctuations may be interpreted such that away from the domain walls, the fields $v_{X}$ have exactly zero fluctuations, i.e., the atoms in this region are in an eigenstate of $n_{l}$. The domain walls instead may display finite density fluctuations but in the thermodynamic limit turn into a region of zero measure. Since the total number and size of droplets is arbitrary, one can construct arbitrarily many steady state field configurations $\partial_{t} \nu_{X}^{(\alpha)}=0$ that have a finite noise averaged density $v^{(\alpha)}=\frac{1}{V} \int_{x}\left\langle v_{X}^{(\alpha)}\right\rangle>0$ and at the same time vanishing fluctuations $\chi^{(\alpha)}=\frac{1}{V} \int_{x}\left\langle\left(v_{X}^{(\alpha)}\right)^{2}\right\rangle-$ $\left(v^{(\alpha)}\right)^{2}=0$ in the thermodynamic limit of infinite system volume $V$, see Fig. 11 for an illustration.

We want to stress that for a flat potential and noise, the coarse-grained Langevin equation becomes linear in $v_{X}$ and thus for any set $\left\{v_{X}^{(\alpha)}\right\}$ of solutions $\partial_{t} v_{X}^{(\alpha)}=0$, a linear combination $v_{X}^{(\vec{a})} \equiv \sum_{\alpha} a_{\alpha} v_{X}^{(\alpha)}$ with $a_{\alpha} \geqslant 0$ and $\sum_{\alpha} a_{\alpha}=1$ is a solution as well. The droplet configurations for different states $\alpha$ do not necessarily overlap in space and thus $\chi^{\vec{a}} \equiv \frac{1}{V} \int_{x}\left\langle\left(v_{X}^{(\vec{a})}\right)^{2}\right\rangle-\left(v^{(\vec{a})}\right)^{2} \geqslant 0$ for average densities $v^{(\vec{a})}=$ $\frac{1}{V} \int_{x}\left\langle v_{X}^{(\vec{a})}\right\rangle$.

While a vanishing noise kernel and a vanishing potential at the coexistence point thus allow us to construct steady states with zero fluctuations, it does by no means enforce that the system relaxes towards such a state. Rather the $v_{X}^{(\alpha)}$ have to be understood as the basis set that spans the manifold of possible steady states. Depending on the specific choice of the $\left\{a_{\alpha}\right\}$, which is set by the initial conditions at $t=0$, these states interpolate between zero and finite fluctuations. The upper bound for the fluctuations is set by the noise kernel in the finite density phase. Connecting the strength of fluctuations with the system's entropy $S$, the coexistence of two phases 
with distinct entropy is thus explained with the coexistence of steady states $v_{X}^{(\vec{a})}$ with distinct fluctuation strength, ranging from strictly zero to a finite value.

This should be contrasted with a coexistence point at a thermal transition, where both the potential and the noise are as well flat but the noise is proportional to the temperature $T$ of the system [1,2]. In principle, this allows us to perform the same construction as above for the steady state manifold but with fluctuations in the droplet states that are bounded from below by the temperature and thus never vanish.

For any large but finite system with volume $V=L^{d}$ where $L$ is the linear dimension, the RG flow is cut off at momenta $k=L^{-1}$. While both $V$ as well as $\chi$ become flat for $k \rightarrow$ 0 , for any $k \sim L^{-1}$ an asymptotically small potential well remains that separates the finite density from the zero density state. As a consequence, no deterministic path connects the two states and transitions between them can only correspond to noise activated trajectories. Since fluctuations on distances $x<L$ have all been integrated out, the only allowed noise activation trajectories are those on distances $x=L$, which have a rate $\gamma_{A} \sim \exp \left(-L^{d} \int_{v_{f}}^{v_{\max }} \frac{V_{k}^{\prime}}{\chi_{k}}\right)$ [5,93], that is exponentially suppressed in the volume and determined by the ratio of $V_{k}^{\prime} / \chi_{k}$ at scale $k=L$. For finite systems, the degeneracy at the coexistence point is thus observably lifted on times $t>\gamma_{A}^{-1}$, which can for instance be associated with the finite system gap of the Lindbladian at the transition.

\section{Experimental signatures}

The striking signature of the first-order phase transition is a discontinuous jump in the steady state Rydberg density $\left\langle v_{X}\right\rangle$ as the system is tuned across the transition. The transition is located at $\Omega_{c}=c_{d} \gamma$, where $c_{d}$ is a numerical factor with $c_{d}=O(1)$ depending on the dimensionality $d$ of the system, i.e., $c_{d=1} \approx 1.3, c_{d=3} \approx 3.8$ for perfect nearest neighbor facilitation. The jump can be observed by measuring the integrated excited state density $n_{R}=\int_{x}\left\langle v_{X}\right\rangle / V$ per volume $V$ and tuning $\Omega$ across $\Omega_{c}$. The jump of $n_{R}$ is $O(1)$ and thus clearly separable from ordinary density fluctuations caused by noise or spatial fluctuations. We showed above that the discontinuity of the density is accompanied with a strong suppression of the noise kernel in the proximity of the transition. The variance of the steady state density $\delta n_{R}=\left(\int_{x}\left\langle v_{X}^{2}\right\rangle / V-n_{R}^{2}\right)=$ $\int_{x} \chi_{k=0} / V$ thus indicates a strong change from Poissonian $\delta n_{R}=\gamma n_{R}$ away from the phase transition to strongly subPoissonian $\delta n_{R} \ll \gamma n_{R}$ at the transition. In contrast, for a constant noise level, i.e., for an equilibrium phase transition, the fluctuations of the steady state density remain constant across the transition. The discontinuity in the steady state density combined with the drastic change in the variance of the density is thus a clear and measurable signature of the first-order dark-state transition. The discontinuous change of the global minimum [bold red line in Fig. 10(a)], which is a significant correction to mean field, is already visible on length scales $x_{k} \sim 10 a$, and thus observable already for very small system sizes.

The formation of droplets as well as the bimodal structure of the noise kernel will be accessible to experiments that can resolve the spatial modulation of the excited state density $\left\langle v_{X}\right\rangle$ as well as its local fluctuations $\left\langle v_{X}^{2}\right\rangle$. The evolution of $V$ and $\chi$ predicts that the formation of droplets, as well as the accompanying, strong density fluctuations that are expressed by the bimodal structure, become observable approximately on scales $10^{2} a \leqslant x_{k} \leqslant 10^{3} a$ [see Figs. 10(a), 10 (c) and 10(d)]. Observing the real space patterns and their fluctuations constitutes a direct proof of the importance of droplets at the dark-state transition, as well as the preferred droplet configuration density, which is indicated by the largest noise level.

A further experimental signature of the nonequilibrium first-order transition is the measurement of dynamic hysteresis $[9,11]$. When the drive strength $\Omega$ is slowly tuned across the coexistence point, the stationary state jumps from the mixed (dark) state to the dark (mixed) state. The relaxation from one state to the other, however, proceeds slowly, leading to a characteristic hysteresis loop for the density $v_{X}$, as a function of initial drive strength $\Omega_{i}$ and time $t$. Although the fRG approach resolves no real time evolution, the Langevin equation in the limit $k \rightarrow 0$ represents an effective equation of motion on macroscopic scales. It predicts a largely asymmetric hysteresis loop, which favors a dynamical transition from the mixed state to the dark state much over the reversed process. On the one hand, the potential $V_{k \rightarrow 0}$ is almost flat in the vicinity of the transition, which leads to a very slow deterministic relaxation of $v_{X}$ towards the true steady state when the system is driven across the transition. The noise kernel $\chi$, on the other hand, is very asymmetric in $v_{X}$, see Fig. 10(c), and thus leads to an average stochastic drift away from the mixed state but does not affect the dark state. This causes a noise induced asymmetry in the hysteresis loop, which is not present for a flat noise profile in thermal equilibrium.

\section{OUTLOOK}

Can the absence of microreversibility, inherent to experiments with driven ultracold atoms, alter many-body correlations and introduce genuine nonequilibrium dynamics on macroscopic scales? We introduced dark-state phase transitions as one paradigmatic, affirmative answer.

We analyzed a corresponding setup, i.e., the transition from statistically mixed to a pure steady state density matrix, in a spin model that is motivated by current experiments on driven dissipative Rydberg ensembles. After identifying a suitable order parameter for this transition, which underlies statistical fluctuations in the mixed state phase but is noiseless in the dark state, we demonstrated that the steady state dynamics of the order parameter can well be analyzed in terms of a nonequilibrium functional renormalization group approach. It allowed us to determine the phase boundary between the dark and the mixed state in the presence of noise and spatial fluctuations on a quantitative level, and to identify the nature of the transition (first or second order). Furthermore, we were able to identify the relevant fluctuations that drive the particular transition in the long-wavelength limit, and to establish the phenomenology for the dark-state transition based on a droplet model and rare fluctuations.

This analysis sheds light on fluctuation induced dynamics close to dark-state transitions and introduces a tool for the 
analysis of general first-order phase transitions, particularly suited for nonthermal setups. From a methodological point of view, one might therefore ask whether the present functional renormalization group approach can be improved to enable, for instance, the analysis of systems driven by a nonMarkovian (quantum) noise.

It also poses a set of new questions concerning quantum dark-state transitions. The identification of a fluctuationless steady state field configuration $v_{X}$ with a dark state in the master equation framework was made concrete for the $v_{X}=0$ ferromagnetic ground state. In order to complete this analogy, one might investigate the coexistence point closer in order to explicitly identify the fluctuationless steady state field configurations $v_{X}^{(\alpha)}$ with another set of pure dark-states $\rho_{\mathrm{SS}}^{(\alpha \geqslant 0)}=$ $\left|\psi_{\alpha}\right\rangle\left\langle\psi_{\alpha}\right|$, that is emergent at the transition, see Fig. 11. One might then ask whether a thermodynamically large number of dark states can exist in a macroscopic many-body system.

The current analysis focuses mainly on the dynamics close to a particular steady state, but is unable to trace the kinetics close to a first-order phase transition, which is in general expected to display universal behavior. This includes the question, in which way the steady state is reached in time and whether one can observe a transient dynamical bistability as predicted from the mean-field picture. Addressing these questions, in addition to the validation of the coexistence of many dark states at the first-order phase transition, are possible tasks for further analysis of the model in terms of a Lindblad master equation approach and, even more directly, in terms of experiments with driven Rydberg ensembles.

\section{ACKNOWLEDGMENTS}

We thank B. Ladewig for fruitful discussions. S.D. and D.R. acknowledge support by the German Research Foundation (DFG) through the Institutional Strategy of the University of Cologne within the German Excellence Initiative (ZUK 81) and S.D. support by the European Research Council via ERC Grant Agreement No. 647434 (DOQS). D.R. is supported, in part, by the NSERC of Canada. M.B. acknowledges support from the Alexander von Humboldt foundation.
[1] K. Binder, Rep. Prog. Phys. 50, 783 (1987).

[2] J. S. Langer, Ann. Phys. (NY) 41, 108 (1967).

[3] F. Letscher, O. Thomas, T. Niederprüm, M. Fleischhauer, and H. Ott, Phys. Rev. X 7, 021020 (2017).

[4] R. Labouvie, B. Santra, S. Heun, and H. Ott, Phys. Rev. Lett. 116, 235302 (2016).

[5] M. Buchhold, B. Everest, M. Marcuzzi, I. Lesanovsky, and S. Diehl, Phys. Rev. B 95, 014308 (2017).

[6] W. Casteels and M. Wouters, Phys. Rev. A 95, 043833 (2017).

[7] W. Casteels, R. Fazio, and C. Ciuti, Phys. Rev. A 95, 012128 (2017).

[8] M. Foss-Feig, P. Niroula, J. T. Young, M. Hafezi, A. V. Gorshkov, R. M. Wilson, and M. F. Maghrebi, Phys. Rev. A 95, 043826 (2017).

[9] S. R. K. Rodriguez, W. Casteels, F. Storme, N. Carlon Zambon, I. Sagnes, L. Le Gratiet, E. Galopin, A. Lemaître, A. Amo, C. Ciuti, and J. Bloch, Phys. Rev. Lett. 118, 247402 (2017).

[10] J. Jin, A. Biella, O. Viyuela, L. Mazza, J. Keeling, R. Fazio, and D. Rossini, Phys. Rev. X 6, 031011 (2016).

[11] A. Angerer, S. Putz, D. O. Krimer, T. Astner, M. Zens, R. Glattauer, K. Streltsov, W. J. Munro, K. Nemoto, S. Rotter, J. Schmiedmayer, and J. Majer, Sci. Adv. 3, e1701626 (2017).

[12] L. Hruby, N. Dogra, M. Landini, T. Donner, and T. Esslinger, Proc. Natl. Acad. Sci. USA 115, 3279 (2018).

[13] A. Le Boité, G. Orso, and C. Ciuti, Phys. Rev. Lett. 110, 233601 (2013).

[14] F. Vicentini, F. Minganti, R. Rota, G. Orso, and C. Ciuti, Phys. Rev. A 97, 013853 (2018).

[15] J. Jin, D. Rossini, M. Leib, M. J. Hartmann, and R. Fazio, Phys. Rev. A 90, 023827 (2014).

[16] L. M. Sieberer, M. Buchhold, and S. Diehl, Rep. Prog. Phys. 79, 096001 (2016).

[17] M. F. Maghrebi and A. V. Gorshkov, Phys. Rev. B 93, 014307 (2016).
[18] S. Diehl, A. Tomadin, A. Micheli, R. Fazio, and P. Zoller, Phys. Rev. Lett. 105, 015702 (2010).

[19] L. M. Sieberer, S. D. Huber, E. Altman, and S. Diehl, Phys. Rev. Lett. 110, 195301 (2013).

[20] L. M. Sieberer, A. Chiocchetta, A. Gambassi, U. C. Täuber, and S. Diehl, Phys. Rev. B 92, 134307 (2015).

[21] M. Marcuzzi, E. Levi, S. Diehl, J. P. Garrahan, and I. Lesanovsky, Phys. Rev. Lett. 113, 210401 (2014).

[22] M. Foss-Feig, J. T. Young, V. V. Albert, A. V. Gorshkov, and M. F. Maghrebi, Phys. Rev. Lett. 119, 190402 (2017).

[23] V. R. Overbeck, M. F. Maghrebi, A. V. Gorshkov, and H. Weimer, Phys. Rev. A 95, 042133 (2017).

[24] H. Weimer, Phys. Rev. Lett. 114, 040402 (2015).

[25] S. Diehl, A. Micheli, A. Kantian, B. Kraus, H. P. Büchler, and P. Zoller, Nat. Phys. 4, 878 (2008).

[26] A. Griessner, A. J. Daley, S. R. Clark, D. Jaksch, and P. Zoller, Phys. Rev. Lett. 97, 220403 (2006).

[27] S. Diehl, E. Rico, M. A. Baranov, and P. Zoller, Nat. Phys. 7, 971 (2011).

[28] F. Verstraete, M. M. Wolf, and J. I. Cirac, Nat. Phys. 5, 633 (2009).

[29] M. Marcuzzi, M. Buchhold, S. Diehl, and I. Lesanovsky, Phys. Rev. Lett. 116, 245701 (2016).

[30] H. K. Janssen, Z. Phys. B 42, 151 (1981).

[31] H.-K. Janssen and U. C. Täuber, Ann. Phys. (NY) 315, 147 (2005).

[32] H. Hinrichsen, Adv. Phys. 49, 815 (2000).

[33] M. Marcuzzi, E. Levi, W. Li, J. P. Garrahan, B. Olmos, and I. Lesanovsky, New J. Phys. 17, 072003 (2015).

[34] H. K. Janssen, K. Oerding, F. van Wijland, and H. J. Hilhorst, Eur. Phys. J. B 7, 137 (1999).

[35] M. Henkel, H. Hinrichsen, and S. Lübeck, Non-Equilibrium Phase Transitions, Theoretical and Mathematical Physics, Vol. 1 (Springer, Berlin, 2009).

[36] H. Hinrichsen, Braz. J. Phys. 30, 69 (2000).

[37] J. Cardy and U. C. Täuber, Phys. Rev. Lett. 77, 4780 (1996). 
[38] U. C. Täuber, in Ageing and the Glass Transition, Lecture Notes in Physics, Vol. 716, edited by M. Henkel, M. Pleimling, and R. Sanctuary (Springer, Berlin, 2007), pp. 295-348.

[39] U. C. Täuber, Critical Dynamics: A Field Theory Approach to Equilibrium and Non-Equilibrium Scaling Behavior (Cambridge University Press, Cambridge, 2014).

[40] J. S. Langer, Ann. Phys. (NY) 54, 258 (1969).

[41] M. A. Muñoz and R. Pastor-Satorras, Phys. Rev. Lett. 90, 204101 (2003).

[42] P. Grassberger, J. Stat. Mech. (2006) P01004.

[43] R. Bidaux, N. Boccara, and H. Chaté, Phys. Rev. A 39, 3094 (1989).

[44] R. Dickman and T. Tomé, Phys. Rev. A 44, 4833 (1991).

[45] L. Giada and M. Marsili, Phys. Rev. E 62, 6015 (2000).

[46] M. M. Valado, C. Simonelli, M. D. Hoogerland, I. Lesanovsky, J. P. Garrahan, E. Arimondo, D. Ciampini, and O. Morsch, Phys. Rev. A 93, 040701 (2016).

[47] C. Wetterich, Phys. Lett. B 301, 90 (1993).

[48] J. Berges, N. Tetradis, and C. Wetterich, Phys. Rep. 363, 223 (2002).

[49] L. Canet, B. Delamotte, O. Deloubrière, and N. Wschebor, Phys. Rev. Lett. 92, 195703 (2004).

[50] S. Finazzi, A. Le Boité, F. Storme, A. Baksic, and C. Ciuti, Phys. Rev. Lett. 115, 080604 (2015).

[51] A. Biella, J. Jin, O. Viyuela, C. Ciuti, R. Fazio, and D. Rossini, Phys. Rev. B 97, 035103 (2018).

[52] F. Lange, Z. Lenarčič, and A. Rosch, Nat. Commun. 8, 15767 (2017).

[53] H. Weimer, M. Müller, I. Lesanovsky, P. Zoller, and H. P. Büchler, Nat. Phys. 6, 382 (2010).

[54] F. Letscher, D. Petrosyan, and M. Fleischhauer, New J. Phys. 19, 113014 (2017).

[55] J. Marino and S. Diehl, Phys. Rev. Lett. 116, 070407 (2016).

[56] E. M. Kessler, G. Giedke, A. Imamoglu, S. F. Yelin, M. D. Lukin, and J. I. Cirac, Phys. Rev. A 86, 012116 (2012).

[57] See Supplemental Material at http://link.aps.org/supplemental/ 10.1103/PhysRevA.98.062117 for this and other exemplary situations, we provide an animated fRG evolution of the deterministic potential, noise kernel and order parameter. We find that the manifold and rather complex results are better conveyed in this way than with individual, static plots only.

[58] S. Bettelli, D. Maxwell, T. Fernholz, C. S. Adams, I. Lesanovsky, and C. Ates, Phys. Rev. A 88, 043436 (2013).

[59] P. Schauß, M. Cheneau, M. Endres, T. Fukuhara, S. Hild, A. Omran, T. Pohl, C. Gross, S. Kuhr, and I. Bloch, Nature (London) 491, 87 (2012).

[60] H. Bernien, S. Schwartz, A. Keesling, H. Levine, A. Omran, H. Pichler, S. Choi, A. S. Zibrov, M. Endres, M. Greiner, V. Vuletić, and M. D. Lukin, Nature (London) 551, 579 (2017).

[61] C. S. Hofmann, G. Günter, H. Schempp, M. Robert-deSaint-Vincent, M. Gärttner, J. Evers, S. Whitlock, and M. Weidemüller, Phys. Rev. Lett. 110, 203601 (2013).

[62] I. Carusotto and C. Ciuti, Rev. Mod. Phys. 85, 299 (2013).

[63] J. F. Poyatos, J. I. Cirac, and P. Zoller, Phys. Rev. Lett. 77, 4728 (1996).

[64] S. Bose, P. L. Knight, M. B. Plenio, and V. Vedral, Phys. Rev. Lett. 83, 5158 (1999).
[65] H. Krauter, C. A. Muschik, K. Jensen, W. Wasilewski, J. M. Petersen, J. I. Cirac, and E. S. Polzik, Phys. Rev. Lett. 107, 080503 (2011).

[66] J. T. Barreiro, M. Müller, P. Schindler, D. Nigg, T. Monz, M. Chwalla, M. Hennrich, C. F. Roos, P. Zoller, and R. Blatt, Nature (London) 470, 486 (2011).

[67] T. E. Lee and M. C. Cross, Phys. Rev. A 85, 063822 (2012).

[68] M. Saffman, T. G. Walker, and K. Mølmer, Rev. Mod. Phys. 82, 2313 (2010).

[69] A. Urvoy, F. Ripka, I. Lesanovsky, D. Booth, J. P. Shaffer, T. Pfau, and R. Löw, Phys. Rev. Lett. 114, 203002 (2015).

[70] T. Amthor, C. Giese, C. S. Hofmann, and M. Weidemüller, Phys. Rev. Lett. 104, 013001 (2010).

[71] R. Gutiérrez, C. Simonelli, M. Archimi, F. Castellucci, E. Arimondo, D. Ciampini, M. Marcuzzi, I. Lesanovsky, and O. Morsch, Phys. Rev. A 96, 041602 (2017).

[72] N. Malossi, M. M. Valado, S. Scotto, P. Huillery, P. Pillet, D. Ciampini, E. Arimondo, and O. Morsch, Phys. Rev. Lett. 113, 023006 (2014).

[73] S. Helmrich, A. Arias, and S. Whitlock, Phys. Rev. A 98, 022109 (2016).

[74] L. Béguin, A. Vernier, R. Chicireanu, T. Lahaye, and A. Browaeys, Phys. Rev. Lett. 110, 263201 (2013).

[75] M. Scully and M. Zubairy, Quantum Optics (Cambridge University Press, Cambridge, 1997).

[76] One should note that $\chi_{\alpha \beta}$ can be equally well derived from a microscopic Hamiltonian, in which the system degrees of freedom are coupled to an appropriate bath that is responsible for the driven-open nature of the system. For the present setup, this has been outlined in Ref. [5]. Here we consider the same setup but focus less on the microscopic implementation and thus refer to the Einstein relation (2.7) instead.

[77] This ensures conservation of the common spin algebra.

[78] This is very similar to the $1 / N$ expansion for large spins performed on each cluster $N_{x}$.

[79] L. Pechenik and H. Levine, Phys. Rev. E 59, 3893 (1999).

[80] C. Ates, B. Olmos, J. P. Garrahan, and I. Lesanovsky, Phys. Rev. A 85, 043620 (2012).

[81] I. Mirgorodskiy, F. Christaller, C. Braun, A. Paris-Mandoki, C. Tresp, and S. Hofferberth, Phys. Rev. A 96, 011402 (2017).

[82] D. Petrosyan, J. Otterbach, and M. Fleischhauer, Phys. Rev. Lett. 107, 213601 (2011).

[83] M. Khasin, B. Meerson, E. Khain, and L. M. Sander, Phys. Rev. Lett. 109, 138104 (2012).

[84] B. Meerson and P. V. Sasorov, Phys. Rev. E 83, 011129 (2011).

[85] R. Dickman, Phys. Rev. E 50, 4404 (1994).

[86] I. Dornic, H. Chaté, and M. A. Muñoz, Phys. Rev. Lett. 94, 100601 (2005).

[87] Y. Tu, G. Grinstein, and M. A. Muñoz, Phys. Rev. Lett. 78, 274 (1997).

[88] A. Rößler, SIAM J. Numer. Anal. 47, 1713 (2009).

[89] M. Wiktorsson, Ann. Appl. Probab. 11, 470 (2001).

[90] P. C. Martin, E. D. Siggia, and H. A. Rose, Phys. Rev. A 8, 423 (1973).

[91] H. K. Janssen, Z. Phys. B 23, 377 (1976).

[92] C. de Dominicis, J. Phys. Colloques (Paris) 37, C1-247 (1976).

[93] A. Kamenev, Field Theory of Non-Equilibrium Systems (Cambridge University Press, Cambridge, 2011). 
[94] L. Canet, J. Phys. A: Math. Gen. 39, 7901 (2006).

[95] L. Canet, H. Chaté, B. Delamotte, I. Dornic, and M. A. Muñoz, Phys. Rev. Lett. 95, 100601 (2005).

[96] M. Buchhold and S. Diehl, Phys. Rev. E 94, 012138 (2016).

[97] I. Boettcher, J. Braun, T. K. Herbst, J. M. Pawlowski, D. Roscher, and C. Wetterich, Phys. Rev. A 91, 013610 (2015).

[98] D. Roscher, J. Braun, and J. E. Drut, Phys. Rev. A 91, 053611 (2015).

[99] J. Berges, N. Tetradis, and C. Wetterich, Phys. Lett. B 393, 387 (1997).

[100] P. Jakubczyk, W. Metzner, and H. Yamase, Phys. Rev. Lett. 103, 220602 (2009).

[101] B. Delamotte, D. Mouhanna, and M. Tissier, Phys. Rev. B 69, 134413 (2004).

[102] L. D. Landau and E. M. Lifshitz, Statistical Physics, Vol. 5 (Butterworth-Heinemann, London, 1980).
[103] H. Hinrichsen, arXiv:cond-mat/0006212.

[104] S. Coleman, Phys. Rev. D 15, 2929 (1977).

[105] Note that this identification can only be made on a qualitative level, since the RG scale $k$ is regulator dependent and not an observable quantity.

[106] This enables us to uniquely identify a first-order phase transition within our RG framework in the first place, as the discontinuity in the value of the order parameter is often not sufficiently distinctive to discriminate between a first- and a steep second-order transition.

[107] While this particular feature is equivalent to what happens at a second-order phase transition, it is here much more complicated to deal with numerically due to the steepness of the noise profile. This is the main reason why the expansion of the dark state in Fig. 10(c) seems to be less pronounced than in Fig. 8(c) where the flow could be run to much smaller scales. 